\title{
Massive Multiuser MIMO in Heterogeneous Cellular Networks with Full Duplex Small Cells
}

\author{
Sunila Akbar, Student Member, IEEE, Yansha Deng, Member, IEEE, Arumugam Nallanathan, Fellow, IEEE, \\ Maged Elkashlan, Member, IEEE, and George K. Karagiannidi, Fellow, IEEE
}

\begin{abstract}
Full duplex (FD) communication has emerged as an attractive solution for increasing the network throughput, by allowing downlink (DL) and uplink (UL) transmissions in the same spectrum. However, only employing FD base stations in heterogeneous cellular networks (HCNs) cause coverage reduction, due to the DL and $U L$ interferences as well as the residual loop interference. We therefore propose $\mathrm{HCNs}$ with half duplex (HD) massive multiuser multiple-input multiple-output (MIMO) macrocell base stations (MBSs) to relax the coverage reduction, and FD small cell base stations (SBSs) to improve spectrum efficiency. A tractable framework of the proposed system is presented, which allows to derive exact and asymptotic expressions for the DL and the UL rate coverage probabilities, and the DL and the UL area spectral efficiencies (ASEs). Monte carlo simulations confirm the accuracy of the analytical results, and it is revealed that equipping massive number of antennas at MBSs enhances the DL rate coverage probability, whereas increasing FD SBSs increases the DL and the UL ASEs. The results also demonstrate that by tuning the $U L$ fractional power control, a desirable performance in both UL and DL can be achieved.
\end{abstract}

Index Terms-Heterogeneous cellular networks, massive multiuser MIMO, full duplex, spectral efficiency, stochastic geometry.

\section{INTRODUCTION}

$\mathbf{T}$ HE emerging fifth-generation (5G) wireless communication system targets higher data rates, roughly 1000 times of the current fourth-generation (4G) system to support exponential increase in wireless data transmissions [1]. In order to meet this target, heterogeneous cellular networks (HCNs) are proposed to boost the network capacity through dense deployment of small cell base stations (SBSs) [2], and multiuser multiple-input multiple-output (MIMO) with large number of antennas at the base station (BS) enables finegrained beamforming towards each mobile user (MU), which brings ultra high throughput [3].

Recently, increasing research has been conducted on fullduplex (FD) communication, which allows transmitting and receiving data simultaneously, within the same frequency band [4]. In theory, FD data transmission is capable of doubling

Manuscript received Nov. 26, 2016; revised May. 30, 2017; accepted June 10, 2017. This work was supported by the U.K. Engineering and Physical Sciences Research Council under Grant EP/M016145/1. The editor coordinating the review of this manuscript and approving it for publication was Prof. Xiqi Gao (Corresponding author: Yansha Deng.).

S. Akbar and Y. Deng are with Department of Informatics, King's College London, London, UK (e-mail: \{sunila.akbar, yansha.deng\}@kcl.ac.uk).

A. Nallanathan and M. Elkashlan are with Queen Mary University of London, London E1 4NS, UK (e-mail:\{arumugam.nallanathan, maged.elkashlan\}@qmul.ac.uk).

G. K. Karagiannidi is with the Department of Electrical and Computer Engineering, Aristotle University of Thessaloniki, 54124 Thessaloniki, Greece (e-mail: geokarag@auth.gr). the spectral efficiency of half-duplex (HD) system. However, FD has been previously regarded as hard to be realized in practice due to its high residual self-interference (SI) problem. Fortunately, the recent advances on SI cancellation, such as antenna separation schemes [5], beamforming-based techniques [6], and digital circuit domain schemes [7], have demonstrated the feasibility of FD transmission for short to medium range wireless communications. For instance, FD transmission can be realized at the access points through shared or separated antenna configurations [8]. In terms of antenna usage, the efficiency of the shared antenna configuration is higher than that of the separated one [9]. Besides, the shared antenna configuration is a promising alternative for separated antenna configuration in short range communications, where the transmit power is low and the antenna isolation requirement is less rigorous compared with medium to long range communication [9]. As a promising candidate for FD technology due to the low transmit power, the SBSs increases network capacity and coverage [10]. As such, leveraging the use of FD technology at the SBSs and massive multiuser MIMO at the macrocell base stations (MBSs) provide a potential solution to improve spectral efficiency of HCNs.

\section{A. Related Work}

1) Multiuser MIMO in HCNs: [11] presented the coverage probability and area spectral efficiency (ASE) for the downlink (DL) MU in HCNs with multiuser MIMO. It was shown that for a given total number of transmit antennas, it is preferable to distribute the antennas across large number of single-antenna BSs rather than small number of multi-antenna BSs. The work in [11] was extended to [12], which studied the load balancing strategy, which maximizes the coverage probability. In [13], it was shown that massive multiuser MIMO BSs and small cells BSs operating in time division duplexing (TDD) mode lead to high area throughput, which can be further improved by installing more BS antennas or deploying more small cells. In [14], a new massive MIMO cognitive radio system was proposed that employs two cognitive radio base stations at the adjacent sides of each cell to realize a full-space spectrum sharing. In [15], a unified UL/DL channel estimation strategy for time division duplex (TDD)/ frequency division duplex (FDD) multiuser massive MIMO systems was presented. In [16], the trade-off between the link reliability and the ASE of HCNs with multiuser MIMO was studied.

2) FD Communication in Cellular Networks: The performance gains brought by FD transmission in cellular networks have been studied in [17-24]. [17] concludes that making different tiers operate in different duplex modes in heterogeneous networks enhances the network throughput. In [18], the 
ASE was derived for small cell networks with FD, and the self-interference (SI) was shown to be dominant compared to the aggregate interference. It is shown in [19] that small cell in-band wireless backhaul has the potential to increase the throughput of massive multiuser MIMO systems. The authors in [20] investigated the spectrum and energy efficiency of the massive MIMO-enabled FD cellular networks. In [21], the rate coverage probability of a massive multiuser MIMOenabled wireless backhaul networks was evaluated, where each SBS can be configured with either in-band or out-of-band FD backhaul mode. In [22], the authors studied the joint in-band backhauling and interference mitigation problem in HCNs, which consists of a massive multiuser MIMO MBS overlaid with self-backhauled small cells. Furthermore, the work in [23] proposed in-band $\alpha$-duplex scheme in multicell networks with FD operation in each cell, which allows a partial overlap between DL and uplink (UL) frequency bands. The results in [23] demonstrated that the overlap parameter, $\alpha$, can be optimized to achieve maximum FD gain. In [24], the cell association problem in multi-tier in-band FD networks was investigated. It is shown that the proposed decoupled cell association, where MUs can be served by different BS in the UL and DL transmission, outperforms the coupled cell association in which MUs associate to the same BS in both DL and UL.

3) Spectral Efficiency and Link Reliability: The two important metrics to evaluate the performance of HCNs, spectral efficiency and outage probability were evaluated in HCNs with wireless power transfer in $[25,26]$. The trade-off between the ASE and the link reliability was discussed in wireless ad-hoc networks [27,28]. In these networks, increasing the density of transmitters affects both link reliability and ASE, therefore the trade-off between them is essential to balance both aspects. The trade-off between the ASE and the coverage probability has been studied in massive multiuser MIMO HCNs [16] and a mixed multi-cell system composed of FD and HD small cells [29].

\section{B. Motivation and Contributions}

The aforementioned literature laid a solid foundation for the feasibility of FD communication in cellular networks. However, operating all the BSs in FD mode is likely to erode the performance gain of the FD communication, since simultaneous DL and UL operations on the same band brings increased interference, and thus reduced coverage. Inspired by the work in [17], where making different tiers operate in different duplex modes in heterogeneous networks enhances the network throughput, we focus on $\mathrm{HCNs}$, where only small cells operate in FD mode, and the macrocells operate in HD mode. We consider FD deployment at the SBSs, due to low transmit power and low mobility of the associated MUs [10]. However, we note that increasing the FD SBSs increases the ASE of the network, they also increase the interference due to the simultaneous DL and UL transmission on the same band which decreases coverage [29]. In order to compensate this cost, a simple solution is to employ massive multiuser MIMO at the BSs. However, due to the facts that: 1) a more powerful
SI cancellation scheme is required to make FD MIMO systems feasible, and 2) the residual interference at each receive chain increase linearly with the number of antennas [30], we have considered massive multiuser MIMO only at the MBSs. For the proposed HCNs, massive antennas at MBSs ensure coverage over large areas, while SBSs act as capacitydrivers [13]. Furthermore, we employ distance-proportional fractional power control in the UL, which provides coverage improvement to the cell-edge MUs and efficient utilization of MUs' battery [31,32].

The main contribution of this work can be summarized as follows:

- We model the $K$-tier HCNs with multiuser MIMO MBSs operating in HD mode and SBSs operating in FD mode. We consider only the DL transmission for MBSs, and both DL and UL transmissions for SBSs. We characterize the network interference generating from the distributed FD SBSs and UL MUs for performance evaluation.

- We derive analytical expressions for the DL rate coverage probability, the DL ASE of the macrocells and small cells, the UL rate coverage probability, and the UL ASE of small cells to evaluate the link reliability and spectral efficiency. To examine the impact of massive multiuser MIMO antennas at the MBS, we derive easy to compute expressions for the asymptotic DL and UL rate coverage probabilities, and asymptotic DL and UL ASEs as the number of antennas at the MBSs grows large.

- Numerical results demonstrate the effectiveness of massive multiuser MIMO at the MBSs and the FD SBSs in enhancing the rate coverage probabilities and the ASEs. Moreover, we show that the distance-proportional fractional power control can be tuned to achieve a desirable performance in both DL and UL, where decreasing the power control factor degrades the UL rate coverage probability, but improves the DL rate coverage probability.

\section{Paper Organization and Notations}

The rest of the paper is organized as follows. In Section II, we discuss the system model of HCNs with multiuser MIMO at the MBSs and FD operation at the SBSs. In Section III, we present the cell association and derive the rate coverage probability and ASE both in the DL and the UL. In Section IV, we evaluate DL and UL rate coverage probabilities and ASEs for the massive multiuser MIMO regime. We present the performance comparison of the proposed HCNs with FD SBSs with the conventional HCNs with HD SBSs in Section V. Finally, numerical results are discussed in Section VI before the paper is concluded in Section VII.

The notations commonly used throughout the paper are presented in Table I.

\section{SySTEM MODEL}

\section{A. Network Model}

We consider $K$-tier HCNs, where the MBSs and the SBSs are spatially located in $\mathbb{R}^{2}$, following homogeneous Poisson point process (HPPP), $\Phi_{b^{\mathrm{M}}}$ and $\Phi_{b^{k}}$ with intensity $\lambda_{b^{\mathrm{M}}}$ and $\lambda_{b^{k}}$ 


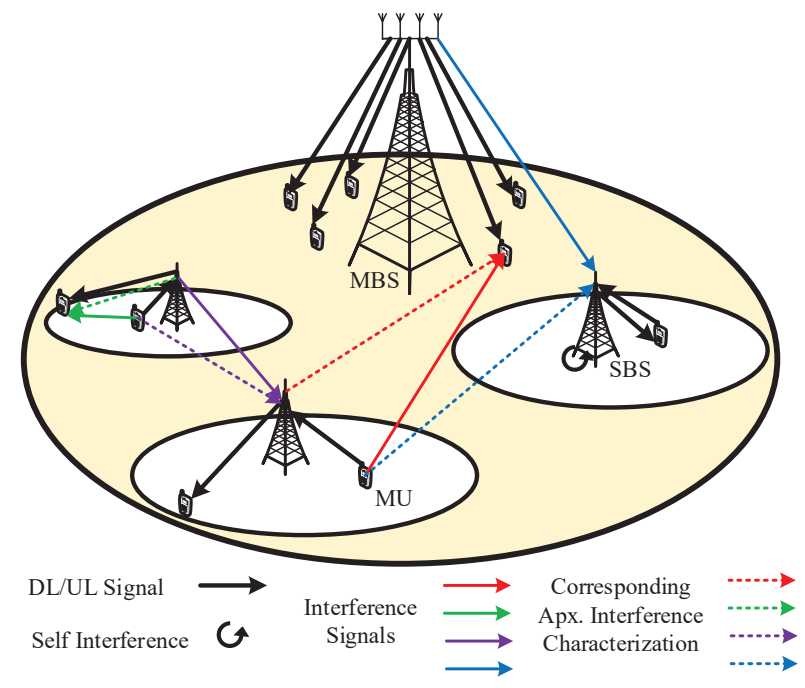

Fig. 1: Example cells of the proposed HCNs with HD multiuser MIMO MBS and FD SBSs and the interference characterizations.

$(k=2, \cdots, K)$, respectively. We consider massive multiuser MIMO at the MBSs, where each BS is equipped with $N$ antennas, serving $U_{\mathrm{M}}$ MUs $\left(1<U_{\mathrm{M}} \ll N\right)$, and operates in HD mode. Each SBS is equipped with single antenna, and is transmitting and receiving at the same time in FD mode [33]. All the MUs have single antenna and operate in HD mode. In this work, we focus on the DL performance of the macrocell without pilot contamination, while the SBSs have transmissions in the DL and UL simultaneously due to the FD operation. The performance of the UL transmission of MBSs can be easily analyzed following our analytical framework, where the scheduled macrocell MUs simultaneously transmit to their serving massive multiuser MBS per resource block in the UL [34]. Linear receive filters are then used for UL signal detection. In HCNs with FD small cells, the DL and UL small cell transmissions occur simultaneously by reusing the spectrum of the DL macrocell transmissions due to the full frequency reuse in HCNs [35]. The network is assumed to be fully-loaded, such that each MBS has $U_{\mathrm{M}}$ active MUs $[11,12]^{1}$, and each SBS serves one active DL MU and one active UL MU in each time instant [24], as shown in Fig. 1. Accordingly, the intensity of the active DL MUs in HCNs is $\lambda_{u}^{\mathrm{DL}}=\left(U_{M} \lambda_{b^{M}}+\sum_{k=1}^{K} \lambda_{b^{k}}\right)$, whereas the active UL MUs per tier are modeled by an independent HPPP $\Phi_{u^{k}}$ with intensity $\lambda_{u^{k}}^{\mathrm{UL}}=\lambda_{b^{k}}$. The analysis will be performed at a typical MU,

\footnotetext{
${ }^{1}$ Here, we limit ourselves to the ideal assumption with the fixed number of $U_{M}$ in each macrocell to ensure equivalent performance at the macrocell MU in each macrocell as in $[36,37]$. We note that in [38], the probability mass function of the number of users served by a generic BS was derived by approximating the area of a Voronoi cell via a gamma-distributed random variable. However, the result in [38] cannot be applied in this paper, since the Euclidean plane is not divided into Voronoi cells based on the considered cell association methods. We highlight that it is an important work to study the case of the flexible $U_{M}$ following a certain distribution in less-dense scenarios.
}

TABLE I: Frequent Notations

\begin{tabular}{c|c}
\hline Notation & Definition \\
\hline \hline$P_{\mathrm{M}}$ & Transmit power of MBS \\
\hline$P_{k}$ & Transmit power of $k$ th tier SBS \\
\hline$\rho_{k}$ & Receiver's sensitivity at the $k$ th tier SBS \\
\hline$\epsilon$ & UL power control factor \\
\hline$\alpha_{\mathrm{M}}$ & Path loss exponent for $k$ th tier small cell \\
\hline$\alpha_{k}$ & Array gain of MBS antenna \\
\hline$G_{a}$ & Frequency dependent constant value \\
\hline$\beta$ & Residual self interfering channel of a $k$ th tier BS \\
\hline$h_{R_{S I}, k}$ & Noise power \\
\hline$N_{o}$ & Gamma function \\
\hline$\Gamma()$. & Gauss hypergeometric function \\
\hline$B_{(.)}[.,]$. &
\end{tabular}

which is assumed to be at the origin.

\section{B. Channel Model}

We model the channel path loss over the distance $|x|$ as $\beta|x|^{-\alpha}$, where $\beta$ is the frequency dependent constant value and $\alpha$ is the path loss exponent. The channels are modeled as independent and identically distributed (i.i.d.) quasi-static Rayleigh fading. We assume TDD mode, where channel reciprocity can be exploited and allows a BS to estimate its DL channels from UL pilots sent by the MUs. Therefore, the resulting number of pilots scales linearly with the number of MUs, and is independent of the number of antennas in contrast to FDD. We consider time division multiple access (TDMA), where several MUs share the same channel in different time slots, thus the BS transmit power is independent of the density of active MUs, and there is no intra-cell interference in each cell. In a snap of time, each MBS can serve $U_{M}$ MUs and each FD SBS serves one DL and one UL MU per channel.

\section{BS and MU Transmit Power Allocation}

Each MBS and SBS transmit with fixed power, $P_{\mathrm{M}}$ and $P_{k}$, respectively. To limit the UL interference and reduce the overall power consumption of MUs, we employ distanceproportional fractional power control [31] in the UL, where the MU at a distance $d$ from the associated SBS adjusts its transmit power with, $P_{u}=\rho_{k} \beta^{-\epsilon} d^{\epsilon \alpha_{j}}$, to compensate largescale fading. Note that, $0 \leq \epsilon \leq 1$, is the power control factor, and $\rho_{k}$ is the receiver sensitivity of the $k$ th tier SBS.

\section{Massive multiuser MIMO}

Each MBS transmit $U_{\mathrm{M}}$ data streams using linear zeroforcing beamforming (ZFBF) with the equal transmit power allocation [39], thus the uncorrelated intra-cell interference is suppressed. We assume sophisticated channel estimation design with sufficient training information that guarantees perfect CSI [40]. In the training phase, each MU sends a pre-assigned orthogonal pilot sequence to the MBS, which is perfectly estimated by the MBS without pilot contamination, therefore the perfect channel state information is available at 
the BSs and MUs. The non-pilot contamination assumption is valid when the pre-assigned pilot sequences used in different macrocells are orthogonal to each other [41]. The maximum number of MUs per MBS depends on the dimension of the UL pilot field. Accordingly, the number of channel vectors that can be estimated and for which the DL precoder can be designed is determined. In our model, we consider fixed number of MUs served by each MBS.

\section{E. Self-Interference Cancellation for FD Small Cells}

The SBS in FD mode receives self-interference from its transmitted signal, and performs self-interference cancellation (self-IC) to combat it. Since, the amount of self-interference depends on the transmit power of the SBS, we define residual self-interference power after self-IC cancellation as [42]

$$
P_{R_{S I}}\left(P_{k}\right)=P_{k}\left|h_{R_{S I}, k}\right|^{2},
$$

where $h_{R_{S I}, k}$ is the residual self interfering channel of a $k$ th tier $\mathrm{BS}$, and $h_{R_{S I}, k}$ is characterized according to the cancellation algorithms. For instance, using digital-domain cancellation algorithms, $h_{R_{S I}, k}$ can be modeled as $h_{R_{S I}, k}=$ $h_{S, k}-\hat{h}_{S, k}$, where $h_{S, k}$ and $\hat{h}_{S, k}$ are the self-interfering channel and its estimate channel, respectively [42]. In [43], $h_{R_{S I}, k}$ is regarded as a constant value with $\left|h_{R_{S I}, k}\right|^{2}=\sigma_{e}^{2}$ for the estimation error variance $\sigma_{e}^{2}$. However, modeling $h_{R_{S I}, k}$ is still challenging for other cancellation techniques, such as analog-domain schemes, propagation-domain schemes, and combined schemes of different domains. The parametrization of the self-IC capability in (1) can make the analysis more generic. Therefore, in our analysis, we consider, a constant value for $h_{R_{S I}, k}{ }^{2}$, given as

$$
\left|h_{R_{S I}, k}\right|^{2}=10^{L_{d B, k} / 10},
$$

where $L_{d B, k}$ is the ratio between the residual self-interference after interference cancellation and the transmit power at the $k$ th tier BS as defined in [17].

\section{F. Cell Association}

To obtain the strongest received signal, we consider the maximum received power cell association rule in the DL transmission, where the DL MU connects to the BS, which provides the maximum long-term average received power [44]. The average received power at a typical DL MU connected to the MBS $m\left(m \in \Phi_{b^{\mathrm{M}}}\right)$ is expressed as

$$
P_{r, \mathrm{M}}=G_{a} \frac{P_{\mathrm{M}}}{U_{\mathrm{M}}} \beta\left|X_{m, u^{\mathrm{M}}}\right|^{-\alpha_{\mathrm{M}}},
$$

where the array gain $G_{a}$ of ZFBF transmission is $N-U_{\mathrm{M}}+1$ [45].

The average received power at a DL MU that is connected to the $k$ th tier SBS $b^{k}\left(b^{k} \in \Phi_{b^{k}}\right)$, is expressed as

$$
P_{r, b^{k}}=P_{k} \beta\left(\left|X_{j, u^{k}}\right|\right)^{-\alpha_{k}} \text {. }
$$

\footnotetext{
${ }^{2}$ The analysis can be easily extended to the case of random $h_{R_{S I}, k}$. For instance, once the probability density function (PDF) of $h_{R_{S I}, k}$ is available for a certain self-IC algorithm, we can average the analytical results derived in the paper over the distribution of $h_{R_{S I}, k}$.
}

We remind that for the UL transmission, the MUs can only associate with the FD SBSs. Considering that the HD UL MU associated to the nearest BS can maximize the UL SINR [24], we consider the nearest BS cell association in the UL.

Based on the cell association model, the set of interfering MUs and FD SBSs may correlate. However, to maintain model tractability, we assume that the set of interfering MUs is independent of the set of interfering FD SBSs as in [18].

\section{G. SINR Models}

1) DL SINR of a Macrocell MU: The signal-tointerference-plus-noise ratio (SINR) for a typical DL macrocell $\mathrm{MU} u_{0}^{\mathrm{M}}$ located at the origin is given as

$$
\mathrm{SINR}_{\mathrm{M}}^{\mathrm{DL}}=\underbrace{\frac{\frac{P_{\mathrm{M}}}{U_{\mathrm{M}}} \beta g_{o, u_{0}^{\mathrm{M}}}\left|X_{o, u_{0}^{\mathrm{M}}}\right|^{-\alpha_{\mathrm{M}}}}{I_{\mathrm{M}, u_{0}^{\mathrm{M}}}+I_{S, u_{0}^{\mathrm{M}}}+I_{u_{u l}^{s}, u_{0}^{\mathrm{M}}}}+N_{0}}_{I_{u_{0}^{\mathrm{M}}}},
$$

where $g_{o, u_{0}^{\mathrm{M}}} \sim \Gamma\left(N-U_{\mathrm{M}}+1,1\right)$ is the small-scale fading channel power gain between the typical DL MU and its serving MBS, and $\left|X_{o, u_{0}^{\mathrm{M}}}\right|$ is the distance between the typical DL MU and its serving MBS. In (5), $I_{\mathrm{M}, u_{0}^{\mathrm{M}}}, I_{S, u_{0}^{\mathrm{M}}}$, and $I_{u_{u l}^{s}, u_{0}^{\mathrm{M}}}$ are the interferences from the other MBSs, the SBSs, and the UL MUs of the small cells given as

$$
\begin{aligned}
I_{\mathrm{M}, u_{0}^{\mathrm{M}}} & =\sum_{x \in \Phi_{b}^{\mathrm{M}} \backslash o} \frac{P_{\mathrm{M}}}{U_{\mathrm{M}}} h_{x, u_{0}^{\mathrm{M}}} \beta\left|X_{x, u_{0}^{\mathrm{M}}}\right|^{-\alpha_{\mathrm{M}}}, \\
I_{S, u_{0}^{\mathrm{M}}} & =\sum_{j=2}^{K} \sum_{y \in \Phi_{b}^{j}} P_{j} h_{y, u_{o}^{\mathrm{M}}} \beta\left|X_{y, u_{0}^{\mathrm{M}}}\right|^{-\alpha_{j}},
\end{aligned}
$$

and

$$
I_{u_{u l}^{s}, u_{0}^{\mathrm{M}}}=\sum_{j=2}^{K} \sum_{z \in \Phi_{u}^{j}} \rho_{j} \beta^{-\epsilon}\left|R_{z, b_{z}}\right|^{\epsilon \alpha_{j}} h_{z, u_{0}^{\mathrm{M}}} \beta\left|X_{z, u_{0}^{\mathrm{M}}}\right|^{-\alpha_{j}},
$$

respectively. In (6), (7), and (8), $h_{x, u_{0}^{\mathrm{M}}} \sim \Gamma\left(U_{\mathrm{M}}, 1\right)$, $h_{y, u_{0}^{\mathrm{M}}} \sim \exp (1)$, and $h_{z, u_{0}^{\mathrm{M}}} \sim \exp (1)$ denote the small-scale fading channel power gains from the MBSs to the typical DL macrocell MU, from the SBSs to the typical DL macrocell MU, and from the UL small cell MUs to the typical DL macrocell MU, respectively, and their corresponding distances are denoted as $\left|X_{x, u_{0}^{\mathrm{M}}}\right|,\left|X_{y, u_{0}^{\mathrm{M}}}\right|$, and $\left|X_{z, u_{0}^{\mathrm{M}}}\right|$, respectively. In (8), $\rho_{j} \beta^{-\epsilon}\left|R_{z, b_{z}}\right|^{\epsilon \alpha_{j}}$ is the transmit power of the UL MU at a distance of $\left|R_{z, b_{z}}\right|$ from its serving SBS, where $\rho_{j}$ is the receiver sensitivity at the SBS of the $j$ th tier and $\epsilon$ is the power control factor.

2) DL SINR of a Small Cell MU: The SINR for a typical DL small cell MU $u_{0}^{k}$ located at the origin can be written as

$$
\mathrm{SINR}_{k}^{\mathrm{DL}}=\frac{P_{k} g_{o, u_{0}^{k}} \beta\left|X_{o, u_{0}^{k}}\right|^{-\alpha_{k}}}{I_{\mathrm{M}, u_{0}^{k}}+I_{S, u_{0}^{k}}+I_{u_{u l}^{s}, u_{0}^{k}}+N_{0}},
$$

where $g_{o, u_{0}^{k}}$ is the small-scale fading channel power gain between the typical DL small cell MU and its serving SBS, and $\left|X_{o, u_{0}^{k}}\right|$ is the distance between the typical DL MU and 
its serving MBS. In (9), $I_{\mathrm{M}, u_{0}^{k}}, I_{S, u_{0}^{k}}$, and $I_{u_{u l}^{s}, u_{0}^{k}}$ are the interference from the other MBSs, the SBSs, and the UL small cell MU, which are given as

$$
\begin{aligned}
I_{\mathrm{M}, u_{0}^{k}} & =\sum_{x \in \Phi_{b}^{\mathrm{M}}} \frac{P_{\mathrm{M}}}{U_{\mathrm{M}}} h_{x, u_{0}^{k}} \beta\left|X_{x, u_{0}^{k}}\right|^{-\alpha_{\mathrm{M}}}, \\
I_{S, u_{0}^{k}} & =\sum_{j=2}^{K} \sum_{y \in \Phi_{b}^{j} \backslash o} P_{j} h_{y, u_{0}^{k}} \beta\left|X_{y, u_{0}^{k}}\right|^{-\alpha_{j}},
\end{aligned}
$$

and

$$
I_{u_{u l}^{s}, u_{0}^{k}}=\sum_{j=2}^{K} \sum_{z \in \Phi_{u}^{j}} \rho_{j} \beta^{-\epsilon}\left|R_{z, b_{z}}\right|^{\epsilon \alpha_{j}} h_{z, u_{0}^{k}} \beta\left|X_{z, u_{0}^{k}}\right|^{-\alpha_{j}},
$$

respectively. In (10), $h_{x, u_{0}^{k}} \sim \Gamma\left(U_{\mathrm{M}}, 1\right)$ denotes the smallscale fading channel power gain between the MBSs and the typical DL small cell MU. The distances between the typical DL small cell MU and the MBSs, the other small cell MU, and the UL small cell MU are denoted as $\left|X_{x, u_{0}^{k}}\right|,\left|X_{y, u_{0}^{k}}\right|$, and $\left|X_{z, u_{0}^{k}}\right|$, respectively.

3) UL SINR of a Small Cell MU: The UL SINR for a typical SBS $b_{0}^{k}$ located at the origin can be written as

$$
\mathrm{SINR}_{k}^{\mathrm{UL}}=\frac{\rho_{k} g_{o, b_{0}^{k}} \beta\left|X_{o, b_{0}^{k}}\right|^{\alpha_{k}(\epsilon-1)}}{P_{R_{S I}}\left(P_{k}\right)+I_{\mathrm{M}, b_{0}^{k}}+I_{S, b_{0}^{k}}+I_{u_{u l}^{s}, b_{0}^{k}}+N_{0}},
$$

where $\rho_{k}$ is the receiver sensitivity of the serving SBS, $g_{o, b_{0}^{k}} \sim$ $\exp (1)$ is the small-scale fading channel power gain between the typical UL small cell MU and its serving SBS, $\left|X_{o, \mathrm{~b}_{0}^{k}}\right|$ is the corresponding distance, and $P_{R_{S I}}\left(P_{k}\right)$ is the residual self-interference power after performing cancellation given in (1). In (13), $I_{\mathrm{M}, b_{0}^{k}}, I_{S, b_{0}^{k}}$, and $I_{u_{u}^{s}, b_{0}^{k}}$ are the interference from the MBSs, the other SBSs, and the other UL small cell MUs given as

$$
\begin{aligned}
I_{\mathrm{M}, b_{0}^{k}} & =\sum_{x \in \Phi_{b}^{\mathrm{M}}} \frac{P_{\mathrm{M}}}{U_{\mathrm{M}}} h_{x, b_{0}^{k}} \beta\left|X_{x, b_{0}^{k}}\right|^{-\alpha_{\mathrm{M}}}, \\
I_{S, b_{0}^{k}} & =\sum_{j=2}^{K} \sum_{y \in \Phi_{b}^{j} \backslash b_{0}^{k}} P_{j} h_{y, b_{0}^{k}} \beta\left|X_{y, b_{0}^{k}}\right|^{-\alpha_{j}},
\end{aligned}
$$

and

$$
I_{u_{u l}^{s}, b_{0}^{k}}=\sum_{j=2}^{K} \sum_{z \in \Phi_{u}^{j} \backslash o} \rho_{j} \beta^{-\epsilon}\left|R_{z, b_{z}}\right|^{\epsilon \alpha_{j}} h_{z, b_{0}^{k}} \beta\left|X_{z, b_{0}^{k}}\right|^{-\alpha_{j}},
$$

respectively. In (14), $h_{x, b_{0}^{k}} \sim \Gamma\left(U_{\mathrm{M}}, 1\right)$ denotes the smallscale fading channel power gain between the typical UL small cell MU and the MBSs. The distances between the typical SBS and the MBSs, the other SBSs, and the other UL small cell MUs are denoted as $\left|X_{x, b_{0}^{k}}\right|,\left|X_{y, b_{0}^{k}}\right|$, and $\left|X_{z, b_{0}^{k}}\right|$, respectively.

\section{H. Interference Characterization}

Characterizing the interference in proposed HCNs is the key challenge in evaluating the system performance. The reason is the difficulty to obtain exact characteristics of the interference from the UL small cell MUs to the DL macrocell MU, $I_{u_{u l}^{s}, u_{0}^{\mathrm{M}}}$ in (8), the interference from the UL small cell MUs to the DL small cell MU, $I_{u_{u l}^{s}, u_{0}^{k}}$ in (12), the interference from the MBSs in the DL to the SBSs in the UL, $I_{\mathrm{M}, b_{0}^{k}}$ in (14), and the interference from the SBSs in the DL to the SBSs in the UL, $I_{S, b_{0}^{k}}$ in (15). We characterize the interferences as shown in Fig. 1 using similar approximation as in [17]. For instance, to characterize $I_{u_{u l}^{s}, u_{0}^{\mathrm{M}}}$, we consider a DL macrocell MU located at $\mathbf{a}$, its serving MBS located at $\mathbf{b}$, a FD SBS located at $\mathbf{c}$, and its associated UL MU at $\mathbf{c}+\mathbf{N}(\mathbf{c})$, where $\mathbf{N}(\mathbf{c})$ is the relative location of small cell MU to its serving SBS at $\mathbf{c}$ in the UL. Generally, the distance between DL macrocell MU a and FD SBS $\mathbf{c}$ is greater than the distance between $\mathbf{c}+\mathbf{N}(\mathbf{c})$ and $\mathbf{c}$, i.e., $\|\mathbf{a}-\mathbf{c}\| \gg\|\mathbf{N}(\mathbf{c})\|$. Therefore, we assume that the distance between macrocell MU at a and UL small cell MU at $\mathbf{c}+\mathbf{N}(\mathbf{c})$ can be approximated as the distance between a macrocell MU at a and the SBS at c. Likewise, we characterize the interferences $I_{u_{u l}^{s}, u_{0}^{k}}, I_{\mathrm{M}, b_{0}^{k}}$, and $I_{S, b_{0}^{k}}$.

\section{Performance Evaluation}

Adjusting the number of antennas at MBSs with multiuser MIMO and the FD SBS densities will affect both the link reliability and ASE of the HCNs. Accordingly, we analyze the performance of the DL and the UL transmission of the HCNs in terms of rate coverage probability and ASE. Since a typical MU can associate with at most one tier, the performance of each tier as well as per tier association probability determine the overall performance of HCNs in the DL and the UL as per the law of total probability. To facilitate the analysis, we first present the per tier association probability.

\section{A. DL Cell Association}

The probability that a typical MU is associated with the MBS is given as in [44], where the transmit powers of the MBSs and SBSs are given in (3) and (4), respectively.

$$
\begin{aligned}
\Lambda_{\mathrm{M}}= & 2 \pi \lambda_{b^{\mathrm{M}}} \int_{0}^{\infty} r \exp \left\{-\pi \lambda_{b^{\mathrm{M}}} r^{2}-\pi \sum_{j=2}^{K} \lambda_{\mathrm{b}^{\mathrm{j}}}\right. \\
& \left.\left(\frac{P_{j}}{\Psi P_{\mathrm{M}}}\right)^{2 / \alpha_{j}} r^{2 \alpha_{\mathrm{M}} / \alpha_{j}}\right\} \mathrm{d} r,
\end{aligned}
$$

where

$$
\Psi=\frac{N-U_{\mathrm{M}}+1}{U_{\mathrm{M}}} .
$$

Similarly, the probability that a typical MU is associated with the $k$ th tier SBS is given as in [44]

$$
\begin{aligned}
\Lambda_{k}^{\mathrm{DL}}= & 2 \pi \lambda_{b^{k}} \int_{0}^{\infty} r \exp \left\{-\pi \sum_{j=2}^{K} \lambda_{b^{j}}\left(P_{j} r^{\alpha_{k}} / P_{k}\right)^{2 / \alpha_{j}}\right. \\
& \left.-\pi \lambda_{b^{\mathrm{M}}}\left(\frac{P_{\mathrm{M}} \Psi}{P_{k}}\right)^{2 / \alpha_{\mathrm{M}}} r^{2 \alpha_{k} / \alpha_{\mathrm{M}}}\right\} \mathrm{dr}
\end{aligned}
$$

where $\Psi$ is given in (18). 


\section{B. UL Cell Association}

In the UL transmission, a typical MUs can only associate with the nearest FD SBS. The probability that a typical MU is associated with the $k$ th tier SBS is given as [44]

$$
\Lambda_{k}^{\mathrm{UL}}=2 \pi \lambda_{b^{k}} \int_{0}^{\infty} r \exp \left\{-\sum_{j=2}^{K} \pi \lambda_{j} r^{2 \alpha_{k} / \alpha_{b j}}\right\} \mathrm{dr} .
$$

\section{DL Rate Coverage Probability}

In this section, we derive the DL rate coverage probability of a typical MU in K-tier HCNs. The DL rate coverage probability of a random MU in the K-tier HCNs is given by

$$
C^{\mathrm{DL}}\left(\mathrm{R}^{\mathrm{DL}}\right)=\Lambda_{\mathrm{M}} C_{\mathrm{M}}\left(\mathrm{R}^{\mathrm{DL}}\right)+\sum_{k=2}^{K} \Lambda_{k}^{\mathrm{DL}} C_{k}^{\mathrm{DL}}\left(\mathrm{R}^{\mathrm{DL}}\right)
$$

where $\Lambda_{\mathrm{M}}$ and $\Lambda_{k}^{\mathrm{DL}}$ are given in (17) and (19), respectively, $C_{\mathrm{M}}\left(\mathrm{R}^{\mathrm{DL}}\right)$ is the $\mathrm{DL}$ rate coverage probability between a typical $\mathrm{MU}$ and its serving $\mathrm{MBS}$, and $C_{k}^{\mathrm{DL}}\left(\mathrm{R}^{\mathrm{DL}}\right)$ is the $\mathrm{DL}$ rate coverage probability between a typical MU and its serving SBS.

In (21), the DL rate coverage probability between a typical MU and its serving MBS is defined as

$C_{\mathrm{M}}\left(\mathrm{R}^{\mathrm{DL}}\right)=\mathbb{E}_{\left|X_{o, u_{0}^{\mathrm{M}}}\right|}\left[\operatorname{Pr}\left[\operatorname{SINR}_{\mathrm{M}}^{\mathrm{DL}}\left(\left|X_{o, u_{0}^{\mathrm{M}}}\right|\right) \geq \gamma^{\mathrm{DL}}|| X_{o, u_{0}^{\mathrm{M}}} \mid\right]\right]$,

where $\operatorname{SINR}_{\mathrm{M}}^{\mathrm{DL}}$ is given in (5) and $\gamma^{\mathrm{DL}}$ is given as

$$
\gamma^{\mathrm{DL}}=\mathrm{e}^{\mathrm{R}^{\mathrm{DL}}}-1 \text {. }
$$

In (23), $\mathrm{R}^{\mathrm{DL}}$ is the DL rate threshold. Similarly, the DL rate coverage probability of typical MU at a distance $\left|X_{o, u_{0}^{k}}\right|$ from its associated SBS in the $k$ th tier is defined as

$C_{k}^{\mathrm{DL}}\left(\mathrm{R}^{\mathrm{DL}}\right)=\mathbb{E}_{\left|X_{o, u_{0}^{k}}\right|}\left[\operatorname{Pr}\left[\operatorname{SINR}_{k}^{\mathrm{DL}}\left(\left|X_{o, u_{0}^{k}}\right|\right) \geq \gamma^{\mathrm{DL}}|| X_{o, u_{0}^{k}} \mid\right]\right]$,

where $\operatorname{SINR}_{\mathrm{k}}^{\mathrm{DL}}$ and $\gamma^{\mathrm{DL}}$ are given in (9) and (23), respectively.

Theorem 1. The DL rate coverage probability of a typical $M U$ associated with the MBS is derived as

$$
\begin{aligned}
C_{\mathrm{M}}\left(\mathrm{R}^{\mathrm{DL}}\right)= & \frac{2 \pi \lambda_{b^{\mathrm{M}}}}{\Lambda_{\mathrm{M}}} \int_{0}^{\infty} x \sum_{n=0}^{N-U_{\mathrm{M}}} \frac{\left(x^{\alpha_{\mathrm{M}}}\right)^{n}}{n !(-1)^{n}} \sum \frac{n !}{\prod_{l=1}^{n} m_{l}(l !)^{m_{l}}} \\
& \kappa(x) \prod_{l=1}^{n}\left(\psi^{(l)}\left(x^{\alpha_{\mathrm{M}}}\right)\right)^{m_{l}} d x
\end{aligned}
$$

where

$$
\begin{aligned}
\kappa(x)= & \exp \left\{-\frac{\gamma^{\mathrm{DL}} U_{\mathrm{M}} q N_{0}}{P_{\mathrm{M}} \beta}-\zeta\left(\frac{\gamma^{\mathrm{DL}} U_{\mathrm{M}} q}{P_{\mathrm{M}} \beta}\right)-\pi \lambda_{b^{\mathrm{M}}} x^{2}\right. \\
& \left.-\pi \sum_{j=2}^{K} \lambda_{b^{k}}\left(\frac{P_{j}}{\Psi P_{\mathrm{M}}}\right)^{\frac{2}{\alpha_{j}}} x^{\frac{2 \alpha_{\mathrm{M}}}{\alpha_{j}}}\right\}
\end{aligned}
$$

$\sum$ is over all $n$-tuples of non-negative integers $\left(m_{1}, \ldots, m_{n}\right)$ that satisfy the constraint $\sum_{l=1}^{n} l . m_{l}=n, \Psi$ is given in (18),
$\Lambda_{\mathrm{M}}$ is given by (17), $\gamma^{\mathrm{DL}}$ is given in (23), and $\zeta(),. \psi^{(1)}($.$) ,$ and $\psi^{(l)}($.$) are given in (27), (28), and (29), respectively, at$ the top of the next page. In (27), (28) and (29), $\lambda_{I_{\Phi_{b j}}}(r)$ is given in (A.8) and $D_{j}^{\mathrm{M}}(x)$ is the distance between the closest interferring $B S$ of the $j$ th tier and the typical macrocell $M U$ given as

$$
D_{j}^{\mathrm{M}}(x)=\left(\frac{P_{j}}{\Psi P_{\mathrm{M}}}\right)^{\frac{1}{\alpha_{j}}} x^{\frac{\alpha_{\mathrm{M}}}{\alpha_{j}}},
$$

where $\Psi$ is given in (18).

Proof. See Appendix A.

Theorem 2. The DL rate coverage probability of a typical $M U$ associated with the kth tier SBS is derived as

$$
\begin{aligned}
& C_{k}^{\mathrm{DL}}\left(\mathrm{R}^{\mathrm{DL}}\right) \\
& =\frac{2 \pi \lambda_{b^{k}}}{\Lambda_{k}^{\mathrm{DL}}} \int_{0}^{\infty} x \exp \left\{-\frac{\gamma^{\mathrm{DL}} x^{\alpha_{k}} N_{0}}{P_{k} \beta}-\Xi(x)-\pi \sum_{j=2}^{K} \lambda_{b^{j}}\right. \\
& \left.\left(\frac{P_{j} x^{\alpha_{k}}}{P_{k}}\right)^{\frac{2}{\alpha_{j}}}-\pi \lambda_{b^{\mathrm{M}}}\left(\frac{P_{\mathrm{M}} \Psi}{P_{k}}\right)^{\frac{2}{\alpha_{\mathrm{M}}}} x^{\frac{2 \alpha_{k}}{\alpha_{\mathrm{M}}}}\right\} d x,
\end{aligned}
$$

where $\Psi, \Lambda_{k}^{\mathrm{DL}}, \gamma^{\mathrm{DL}}$, and $\Xi($.$) are given in (18), (19), and$ (23), and (32), respectively. In (32), $\lambda_{I_{\Phi_{b j}}}(r)$ is given in (A.8), $D_{\mathrm{M}}^{k}(x)$ is the distance between the closest interfering MBS and the typical small cell $M U$, and $D_{j}^{k}(x)$ is the distance between the closest interfering BS in the jth tier and the typical small cell $M U$, given as

$$
D_{\mathrm{M}}^{k}(x)=\left(\frac{\Psi P_{\mathrm{M}}}{P_{k}}\right)^{\frac{1}{\alpha_{\mathrm{M}}}} x^{\frac{\alpha_{k}}{\alpha_{\mathrm{M}}}}
$$

and

$$
D_{j}^{k}(x)=\left(\frac{P_{j}}{P_{k}}\right)^{\frac{1}{\alpha_{j}}} x^{\frac{\alpha_{k}}{\alpha_{j}}} .
$$

In (33), $\Psi$ is given in (18).

Proof. The proof follows analogous steps to Theorem 1.

\section{DL Area Spectral Efficiency}

The DL ASE measures the capacity of HCNs in the DL defined by $[11,46]$. In this section, we define the DL ASE of the proposed model as

$$
\begin{aligned}
\mathrm{ASE}^{\mathrm{DL}} & =\lambda_{b^{\mathrm{M}}} U_{\mathrm{M}} C_{\mathrm{M}}\left(\mathrm{R}^{\mathrm{DL}}\right) \ln \left(1+\gamma^{\mathrm{DL}}\right) \\
& +\sum_{k=2}^{K} \lambda_{b^{k}} C_{k}^{\mathrm{DL}}\left(\mathrm{R}^{\mathrm{DL}}\right) \ln \left(1+\gamma^{\mathrm{DL}}\right),
\end{aligned}
$$

where $C_{\mathrm{M}}\left(\mathrm{R}^{\mathrm{DL}}\right), C_{k}^{\mathrm{DL}}\left(\mathrm{R}^{\mathrm{DL}}\right)$, and $\gamma^{\mathrm{DL}}$ are given in (25), (31), and (23), respectively.

In the following, we present the UL performance metrics which reflect the effect of the self-IC, the density of SBSs, the transmit power of SBSs, and the power control on the UL performance in the $\mathrm{HCN}$. We characterize the UL performance in terms of the UL rate coverage probability and the UL ASE. 


$$
\begin{aligned}
& \zeta(s)=2 \pi \lambda_{b^{\mathrm{M}}} \sum_{\nu=1}^{U_{\mathrm{M}}}\left(\begin{array}{c}
U_{\mathrm{M}} \\
\nu
\end{array}\right)\left(\frac{P_{\mathrm{M}}}{U_{\mathrm{M}}} \beta\right)^{\nu} s^{\nu} \frac{\left(-s \frac{P_{\mathrm{M}}}{U_{\mathrm{M}}} \beta\right)^{-\nu+\frac{2}{\alpha_{\mathrm{M}}}}}{\alpha_{\mathrm{M}}} B\left(-s \frac{P_{\mathrm{M}}}{U_{\mathrm{M}}} \beta x^{-\alpha_{\mathrm{M}}}\right)\left[\nu-\frac{2}{\alpha_{\mathrm{M}}}, 1-U_{\mathrm{M}}\right]+\sum_{j=2}^{K} 2 \pi \lambda_{b^{j}}\left\{s P_{j} \beta\right. \\
& \frac{D_{j}^{\mathrm{M}}(x)^{2-\alpha_{j}}}{\alpha_{j}-2}{ }_{2} F_{1}\left[\frac{\alpha_{j}-2}{\alpha_{j}}, 1 ; 2-\frac{2}{\alpha_{j}} ;-s P_{j} \beta\left(D_{j}^{\mathrm{M}}(x)\right)^{-\alpha_{j}}\right]+\int_{0}^{\infty} \int_{0}^{r^{2}} \frac{1}{1+\left(s \rho_{j} \beta^{(1-\epsilon)}\right)^{-1} u^{-\alpha_{j} \epsilon / 2} r^{\alpha_{j}}} \\
& \left.\left(\pi \sum_{j=2}^{K} \lambda_{b^{j}} e^{-\pi \sum_{j=2}^{K} \lambda_{b^{j}} u}\right) \lambda_{I_{\Phi^{\mathrm{UL}}}^{\mathrm{U}}}(r) \mathrm{d} u r \mathrm{~d} r\right\} \\
& \psi^{(1)}(q)=-\frac{\gamma^{\mathrm{DL}} U_{\mathrm{M}} N_{0}}{P_{\mathrm{M}} \beta}-2 \pi \lambda_{b^{\mathrm{M}}} U_{\mathrm{M}} \gamma^{\mathrm{DL}} \frac{x^{2-\alpha_{\mathrm{M}}}}{\alpha_{\mathrm{M}}-2}{ }_{2} F_{1}\left[\frac{\alpha_{\mathrm{M}}-2}{\alpha_{\mathrm{M}}}, U_{\mathrm{M}}+1 ; 2-\frac{2}{\alpha_{\mathrm{M}}} ;-\gamma^{\mathrm{DL}} q x^{-\alpha_{\mathrm{M}}}\right]-\sum_{j=2}^{K} 2 \pi \lambda_{b^{j}}\left\{\frac{\gamma^{\mathrm{DL}} U_{\mathrm{M}} P_{j}}{P_{\mathrm{M}}}\right. \\
& \frac{D_{j}^{\mathrm{M}}(x)^{2-\alpha_{j}}}{\alpha_{j}-2}{ }_{2} F_{1}\left[\frac{\alpha_{j}-2}{\alpha_{j}}, 2 ; 2-\frac{2}{\alpha_{j}} ;-\frac{\gamma^{\mathrm{DL}} U_{\mathrm{M}} P_{j} q}{P_{\mathrm{M}}}\left(D_{j}^{\mathrm{M}}(x)\right)^{-\alpha_{j}}\right]-\int_{0}^{\infty} \int_{0}^{r^{2}}\left(\frac{P_{\mathrm{M}} \beta^{\epsilon} u^{-\alpha_{j} \epsilon / 2} r^{\alpha_{j}}}{\gamma^{\mathrm{DL}} U_{\mathrm{M}} \rho}\right)\left(1+\frac{P_{\mathrm{M}} \beta^{\epsilon} u^{-\alpha_{j} \epsilon / 2} r^{\alpha_{j}}}{\gamma^{\mathrm{DL}} U_{\mathrm{M}} \rho}\right)^{-2} \\
& \left.q^{-2}\left(\pi \sum_{j=2}^{K} \lambda_{b^{j}} e^{-\pi \sum_{j=2}^{K} \lambda_{b^{j}} u}\right) \lambda_{I_{\Phi_{b j}}}(r) \mathrm{d} u r \mathrm{~d} r\right\} \\
& \psi^{(l)}(q)=2 \pi \lambda_{b^{\mathrm{M}}} \frac{\left(U_{\mathrm{M}}+l-1\right) !}{\left(U_{\mathrm{M}}-1\right) !}\left(-\gamma^{\mathrm{DL}}\right)^{\frac{2}{\alpha_{\mathrm{M}}}} \frac{(q)^{-l+\frac{2}{\alpha_{\mathrm{M}}}}}{\alpha_{\mathrm{M}}} B_{\left(-\gamma^{\mathrm{DL}} q x^{-\alpha_{\mathrm{M}}}\right)}\left[l-\frac{2}{\alpha_{\mathrm{M}}}, 1-U_{\mathrm{M}}-l\right]+\sum_{j=2}^{K} 2 \pi \lambda_{b^{j}}\left\{l !\left(-\frac{\gamma^{\mathrm{DL}} U_{\mathrm{M}} P_{j}}{P_{\mathrm{M}}}\right)^{\frac{2}{\alpha_{\mathrm{M}}}}\right. \\
& \frac{(q)^{-l+\frac{2}{\alpha_{\mathrm{M}}}}}{\alpha_{\mathrm{M}}} B\left(-\frac{\gamma^{\mathrm{DL}} U_{\mathrm{M}} P_{j} q}{P_{\mathrm{M}}}\left(D_{j}^{\mathrm{M}}(x)\right)^{-\alpha_{\mathrm{M}}}\right)\left[l-\frac{2}{\alpha_{\mathrm{M}}},-l\right]-\int_{0}^{\infty} \int_{0}^{r^{2}}\left(\frac{P_{\mathrm{M}} \beta^{\epsilon} u^{-\alpha_{j} \epsilon / 2} r^{\alpha_{j}}}{\gamma^{\mathrm{DL}} U_{\mathrm{M}} \rho}\right) \prod_{i=2}^{l}(-i)\left(\frac{P_{\mathrm{M}} \beta^{\epsilon} u^{-\alpha_{j} \epsilon / 2} r^{\alpha_{j}}}{\gamma^{\mathrm{DL}} U_{\mathrm{M}} \rho}+q\right)^{-(l+1)} \\
& \left.\left(\pi \sum_{j=2}^{K} \lambda_{b^{j}} e^{-\pi \sum_{j=2}^{K} \lambda_{b^{j}} u}\right) \lambda_{I_{\Phi_{b j}^{\mathrm{UL}}}}(r) \mathrm{d} u r \mathrm{~d} r\right\}
\end{aligned}
$$

$$
\begin{aligned}
\Xi(x)= & 2 \pi \lambda_{b^{\mathrm{M}}} \sum_{\nu=1}^{U_{\mathrm{M}}}\left(\begin{array}{c}
U_{\mathrm{M}} \\
\nu
\end{array}\right)\left(\frac{\gamma^{\mathrm{DL}} x^{\alpha_{k}} P_{\mathrm{M}}}{P_{k} U_{\mathrm{M}}}\right)^{\nu} \frac{\left(-\frac{\gamma^{\mathrm{DL}} x^{\alpha_{k} P_{\mathrm{M}}}}{P_{k} U_{\mathrm{M}}}\right)^{-\nu+\frac{2}{\alpha_{\mathrm{M}}}}}{\alpha_{\mathrm{M}}} B\left(-\frac{\gamma^{\mathrm{DL}} x^{\alpha_{k} P_{\mathrm{M}}}}{P_{k} U_{\mathrm{M}}}\left(D_{\mathrm{M}}^{k}(x)\right)^{-\alpha_{\mathrm{M}}}\right)\left[\nu-\frac{2}{\alpha_{\mathrm{M}}}, 1-U_{\mathrm{M}}\right] \\
& +\sum_{j=2}^{K} 2 \pi \lambda_{b^{j}}\left\{\gamma^{\mathrm{DL}} x^{\alpha_{k}} \frac{P_{j}}{P_{k}} \frac{\left(D_{j}^{k}(x)\right)^{2-\alpha_{j}}}{\left(\alpha_{j}-2\right)}{ }_{2} F_{1}\left[\frac{\alpha_{j}-2}{\alpha_{j}}, 1 ; 2-\frac{2}{\alpha_{j}} ;-\frac{\gamma^{\mathrm{DL}} P_{j}}{P_{k}}\left(D_{j}^{k}(x)\right)^{\alpha_{k}-\alpha_{j}}\right]\right. \\
& \left.\left.+\int_{0}^{\infty} \int_{0}^{r^{2}} \frac{1}{1+\left(\frac{\gamma^{\mathrm{DL}} x^{\alpha} k}{P_{k} \beta} \rho_{j} \beta^{(1-\epsilon)}\right)^{-1} u^{-\alpha_{j} \epsilon / 2} r^{\alpha_{j}}} \lambda_{j=2} e^{-\pi \sum_{j=2}^{K} \lambda_{b j} u}\right) \lambda_{I_{\Phi} \mathrm{UL}}(r) \mathrm{d} u r \mathrm{~d} r\right\}
\end{aligned}
$$

\section{E. UL Rate Coverage Probability}

In this section, we derive the UL rate coverage probability using

$$
C^{\mathrm{UL}}\left(\mathrm{R}^{\mathrm{UL}}\right)=\sum_{k=2}^{K} \Lambda_{k}^{\mathrm{UL}} C_{k}^{\mathrm{UL}}\left(\mathrm{R}^{\mathrm{UL}}\right)
$$

where $\Lambda_{k}^{\mathrm{UL}}$ is given in (20), and $C_{k}^{\mathrm{UL}}\left(\mathrm{R}^{\mathrm{UL}}\right)$ is the $\mathrm{UL}$ rate coverage probability between a typical MU and its serving SBS defined as

$$
\begin{aligned}
C_{k}^{\mathrm{UL}}\left(\mathrm{R}^{\mathrm{UL}}\right)= & \mathbb{E}_{\left|X_{o, b_{0}^{k}}\right|}\left[\operatorname { P r } \left[\operatorname{SINR}_{k}^{\mathrm{UL}}\left(\left|X_{o, b_{0}^{k}}\right|\right)\right.\right. \\
& \left.\left.\geq \gamma^{\mathrm{UL}}||\left|X_{o, b_{0}^{k}}\right| \mid\right]\right]
\end{aligned}
$$

where $\operatorname{SINR}_{\mathrm{k}}^{\mathrm{UL}}$ is given in (13) and $\gamma^{\mathrm{UL}}$ is given as

$$
\gamma^{\mathrm{UL}}=\mathrm{e}^{\mathrm{R}^{\mathrm{UL}}}-1
$$

and $\mathrm{R}^{\mathrm{UL}}$ is the $\mathrm{UL}$ rate threshold.

Theorem 3. The UL rate coverage probability of a typical $M U$ associated with the kth tier SBS is derived as

$$
\begin{aligned}
& C_{k}^{\mathrm{UL}}\left(\mathrm{R}^{\mathrm{UL}}\right) \\
= & \frac{2 \pi \lambda_{b^{k}}}{\Lambda_{k}^{\mathrm{UL}}} \int_{0}^{\infty} x \exp \left\{-\frac{\gamma^{\mathrm{UL}} x^{\alpha_{k}}\left(N_{0}+P_{k}\left|h_{R_{S I}, k}\right|^{2}\right)}{\left(\rho_{k} \beta^{-\epsilon} d^{\epsilon \alpha_{j}}\right) \beta}\right. \\
& \left.-\Upsilon(x)-\sum_{j=2}^{K} \pi \lambda_{b^{j}}\left(P_{j} / P_{k}\right)^{2 / \alpha_{j}} x^{2 \alpha_{k} / \alpha_{j}}\right\} d x,
\end{aligned}
$$

where $\Lambda_{k}^{\mathrm{UL}}, \gamma^{\mathrm{UL}}$, and $\left|h_{R_{S I}, k}\right|^{2}$ are given in (20), (38) and (2), respectively. In (39), $\Upsilon($.$) is given in (40) at the top of$ the next page. 


$$
\begin{aligned}
\Upsilon(x)= & 2 \pi \lambda_{b^{\mathrm{M}}} \sum_{\nu=1}^{U_{\mathrm{M}}}\left(\begin{array}{c}
U_{\mathrm{M}} \\
\nu
\end{array}\right)\left(\frac{\gamma^{\mathrm{UL}} x^{\alpha_{k}} P_{\mathrm{M}}}{\rho_{k} x^{\alpha_{k} \epsilon} \beta^{-\epsilon} U_{\mathrm{M}} \alpha_{\mathrm{M}}}\right) \frac{\Gamma\left(\nu-\frac{2}{\alpha_{\mathrm{M}}}\right) \Gamma\left(U_{\mathrm{M}}-\nu+\frac{2}{\alpha_{\mathrm{M}}}\right)}{\Gamma\left(U_{\mathrm{M}}\right)}+\sum_{j=2}^{K} 2 \pi \lambda_{b^{j}}\left(\frac{\gamma^{\mathrm{UL}} P_{j} x^{\alpha_{k}}}{\rho_{k} x^{\alpha_{k} \epsilon} \beta^{-\epsilon}}\right. \\
& \frac{x^{2-\alpha_{j}}}{\left(\alpha_{j}-2\right)}{ }_{2} F_{1}\left[1,1-\frac{2}{\alpha_{j}} ; 2-\frac{2}{\alpha_{j}} ;-\frac{\gamma^{\mathrm{UL}} P_{j}}{\rho_{k} x^{\alpha_{k} \epsilon} \beta^{-\epsilon}} x^{\left(\alpha_{k}-\alpha_{j}\right)}\right]+\int_{0}^{\infty} \int_{0}^{r^{2}} \frac{1}{1+\left(\frac{\gamma_{\mathrm{UL}} x^{(1-\epsilon) \alpha_{k}}}{\rho_{k}} \rho_{j}\right)^{-1} u^{-\alpha_{j} \epsilon / 2} r^{\alpha_{j}}} \\
& \left.\left(\pi \sum_{j=2}^{K} \lambda_{b^{j}} e^{-\pi \sum_{j=2}^{K} \lambda_{b^{j}} u}\right) \mathrm{d} u r \mathrm{~d} r\right\} .
\end{aligned}
$$

Proof. The proof follows analogous steps to Theorem 1.

\section{F. UL Area Spectral Efficiency}

In this section, we derive the UL ASE in the K-tier HCNs. The UL ASE measures the capacity of HCNs in the UL, given as

$$
\mathrm{ASE}^{\mathrm{UL}}=\sum_{k=2}^{K} \lambda_{b^{k}} C_{k}^{\mathrm{UL}}\left(\mathrm{R}^{\mathrm{UL}}\right) \ln \left(1+\gamma^{\mathrm{UL}}\right),
$$

where $C_{k}^{\mathrm{UL}}\left(\mathrm{R}^{\mathrm{UL}}\right)$ and $\gamma^{\mathrm{UL}}$ are given in (39) and (38), respectively.

\section{Asymptotic Performance Evaluation: Massive MULTIUSER MIMO REGIME}

In this section, we analyze the asymptotic performance of $K$-tier HCNs in which MBSs are equipped with massive multiuser MIMO antennas. The large number of antennas focusses energy into ever smaller regions of space to bring huge improvements in throughput and energy efficiency. We refer to the massive multiuser MIMO regime as the case where $1<U_{\mathrm{M}} \ll N$.

\section{A. SINR Models}

1) DL SINR of a Macrocell MU: With massive multiuser MIMO at the MBSs, the SINR for a typical DL macrocell MU defined in (5) can be simplified to

$$
\left.\mathrm{SINR}_{\mathrm{M}}^{\mathrm{DL}}\right|_{m \mathrm{M}}=\underbrace{\frac{P_{\mathrm{M}} \beta\left|X_{o, u_{0}^{\mathrm{M}}}\right|^{-\alpha_{\mathrm{M}}}}{I_{\mathrm{M}_{m \mathrm{M}}, u_{0}^{\mathrm{M}}}+I_{S, u_{0}^{\mathrm{M}}}+I_{u_{u l}^{s}, u_{0}^{\mathrm{M}}}}+N_{0}}_{\left.I_{u_{o}^{\mathrm{M}}}\right|_{m \mathrm{M}}},
$$

where the massive multiuser MIMO gain, $N-U_{\mathrm{M}}+$ 1 , and the impact of equal power allocation per backhaul stream (i.e., the denominator of MBS's transmit power $\left.\frac{P_{\mathrm{M}}}{U_{\mathrm{M}}}\right)$ have already been incorporated in (45). In

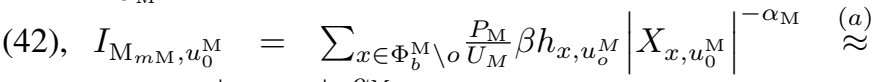
$\sum_{x \in \Phi_{b}^{\mathrm{M}} \backslash o} P_{\mathrm{M}} \beta\left|X_{x, u_{0}^{\mathrm{M}}}\right|^{-\alpha_{\mathrm{M}}}$, the approximation in (a) results due to the fact that with the large number of $U_{\mathrm{M}}$, i.e., $\left(1<U_{\mathrm{M}} \ll N\right)$, the small scale channel fading vanish by the channel hardening effect as in [21]. In (42), $I_{S, u_{0}^{\mathrm{M}}}$ and $I_{u_{u l}^{s}, u_{0}^{\mathrm{M}}}$ are given in (7) and (8), respectively.
2) DL SINR of a Small Cell MU: For the massive multiuser MIMO, the DL SINR for a typical small cell MU defined in (9) can be given as

$$
\left.\mathrm{SINR}_{k}^{\mathrm{DL}}\right|_{m \mathrm{M}}=\frac{P_{k} g_{o, u_{0}^{k}} \beta\left|X_{o, u_{0}^{k}}\right|^{-\alpha_{k}}}{I_{\mathrm{M}_{m \mathrm{M}}, u_{0}^{k}}+I_{S, u_{0}^{k}}+I_{u_{u l}^{s}, u_{0}^{k}}+N_{0}},
$$

where $I_{\mathrm{M}_{m \mathrm{M}}, u_{0}^{k}}=\sum_{x \in \Phi_{b}^{\mathrm{M}}} \frac{P_{\mathrm{M}}}{U_{\mathrm{M}}} \beta\left|X_{x, u_{0}^{k}}\right|^{-\alpha_{\mathrm{M}}}$, i.e., no shortterm fading factor due to channel hardening effect. In (43), $I_{S, u_{0}^{k}}$ and $I_{u_{u}^{s}, u_{0}^{k}}$ are given in (11) and (12), respectively.

3) UL SINR of a Small Cell MU: For the massive multiuser MIMO case, the UL SINR for a typical SBS given in (13) can be written as

$$
\begin{aligned}
& \left.\operatorname{SINR}_{k}^{\mathrm{UL}}\right|_{m \mathrm{M}} \\
= & \frac{\rho_{k} g_{o, b_{0}^{k}} \beta\left|X_{o, b_{0}^{k}}\right|^{\alpha_{k}(\epsilon-1)}}{P_{R_{S I}}\left(P_{k}\right)+I_{\mathrm{M}_{m \mathrm{M}}, b_{0}^{k}}+I_{S, b_{0}^{k}}+I_{u_{u l}^{s}, b_{0}^{k}}+N_{0}},
\end{aligned}
$$

where $I_{\mathrm{M}_{m \mathrm{M}}, b_{0}^{k}}=\sum_{x \in \Phi_{b}^{\mathrm{M}}} \frac{P_{\mathrm{M}}}{U_{\mathrm{M}}} \beta\left|X_{x, b_{0}^{k}}\right|^{-\alpha_{\mathrm{M}}}$, i.e., no shortterm fading factor due to channel hardening effect. In (44), $P_{R_{S I}}\left(P_{k}\right), I_{S, b_{0}^{k}}$, and $I_{u_{u l}^{s}, b_{0}^{k}}$ are given in (1), (11) and (12), respectively.

\section{B. Asymptotic DL Rate Coverage Probability}

In this analysis, we use the following formula for the DL rate coverage probability of the macrocell with massive multiuser MIMO [47]

$$
\begin{aligned}
\left.C_{\mathrm{M}}\left(\mathrm{R}^{\mathrm{DL}}\right)\right|_{m \mathrm{M}}= & \mathbb{E}_{\left|X_{o, u_{0}^{\mathrm{M}}}\right|}\left[\mathbb { E } _ { \operatorname { S I N R } _ { \mathrm { M } } ^ { \mathrm { DL } } } \left[\operatorname { P r } \left[\operatorname{SINR}_{\mathrm{M}}^{\mathrm{DL}}\left(\left|\mathrm{X}_{\mathrm{o}, \mathrm{u}_{0}^{\mathrm{M}}}\right|\right)\right.\right.\right. \\
& \left.\left.\geq \gamma_{\mathrm{M}_{m \mathrm{M}}}^{\mathrm{DL}}|| X_{o, u_{0}^{\mathrm{M}}} \mid\right]\right]
\end{aligned}
$$

where

$$
\gamma_{\mathrm{M}_{m \mathrm{M}}}^{D L}=\frac{\gamma^{\mathrm{DL}}}{\Psi}
$$

and $\gamma^{\mathrm{DL}}$ and $\Psi$ are given in (23) and (18), respectively. The DL and UL rate coverage probabilities definitions for the small cells in the massive multiuser MIMO case will be the same as in the multiuser MIMO case, which are defined as (24), and (37), respectively.

We present the asymptotic DL rate coverage probability of a typical MU associated with the MBS and the $k$ th tier SBS in Theorem 4 and Theorem 5, respectively. 
Theorem 4. For the massive multiuser MIMO regime, the $D L$ rate coverage probability of a typical MU associated with the $M B S$ is derived as

$$
\begin{aligned}
& \left.C_{\mathrm{M}}\left(\mathrm{R}^{\mathrm{DL}}\right)\right|_{m \mathrm{M}} \\
& =\frac{2 \pi \lambda_{b^{\mathrm{M}}}}{\Lambda_{\mathrm{M}}} \int_{0}^{\infty} x\left[\frac{1}{2}-\frac{1}{\pi} \int_{0}^{\infty} \operatorname{Im}\left[\operatorname { e x p } \left\{-\chi_{1}(x, \mathrm{w})\right.\right.\right. \\
& -\pi \lambda_{b^{\mathrm{M}}} \chi_{2}(x, \mathrm{w})-\sum_{j=2}^{K} 2 \pi \lambda_{b^{j}}\left\{\chi_{3}(x, \mathrm{w})+\chi_{4}(x, \mathrm{w})\right\}- \\
& \left.\left.\left.\pi \lambda_{b^{\mathrm{M}}} x^{2}-\pi \sum_{j=2}^{K} \lambda_{b^{k}}\left(\frac{P_{j}}{\Psi P_{\mathrm{M}}}\right)^{2 / \alpha_{j}} r^{2 \alpha_{\mathrm{M}} / \alpha_{j}}\right\}\right]\right] \frac{\mathrm{dw}}{\mathrm{w}} \mathrm{d} x,
\end{aligned}
$$

where

$$
\begin{aligned}
\chi_{1}(x, \mathrm{w})= & \mathrm{jw}\left(\frac{P_{\mathrm{M}} \beta}{\gamma_{\mathrm{M}_{m \mathrm{M}} L}^{D L} x^{\alpha_{\mathrm{M}}}}-N_{o}\right) \\
\chi_{2}(x, \mathrm{w})= & \frac{\Gamma\left(1-\frac{2}{\alpha_{\mathrm{M}}}\right)+\frac{2}{\alpha_{\mathrm{M}}} \Gamma_{u}\left(-\frac{2}{\alpha_{\mathrm{M}}}, \frac{-\mathrm{jw} P_{\mathrm{M}} \beta}{x^{\alpha_{\mathrm{M}}}}\right)}{\left(-\mathrm{jw} P_{\mathrm{M}} \beta\right)^{\frac{2}{\alpha_{\mathrm{M}}}}}-x^{2}, \\
\chi_{3}(x, \mathrm{w})= & \left(\frac{P_{j}^{\alpha_{j} / 2}(-\mathrm{jw}) \beta\left(\frac{x^{\alpha_{\mathrm{M}}}}{\Psi P_{\mathrm{M}}}\right)^{2 / \alpha_{j}-1}}{\alpha_{j}-2}\right) \\
\chi_{4}(x, \mathrm{w})= & \int_{0}^{\infty} \int_{0}^{r_{1}}\left[1,1-\frac{2}{\alpha_{j}} ; 2-\frac{2}{\alpha_{j}} ; \frac{\mathrm{jw} \Psi P_{\mathrm{M}}}{x^{\alpha_{\mathrm{M}}}}\right] \\
& \left(\pi \sum_{j=2}^{K} \lambda_{b^{j}} e^{-\pi \sum_{j=2}^{K} \lambda_{b j} u}\right) \lambda_{I_{\Phi^{\mathrm{UL}}}}(r) \mathrm{d} u r \mathrm{~d} r
\end{aligned}
$$

and $\Lambda_{\mathrm{M}}, \gamma_{\mathrm{M}_{m \mathrm{M}}}^{\mathrm{DL}}, \Psi$, and $\lambda_{I_{\Phi_{\mathrm{SU}}}}(r)$ are given in (17), (46), (18), and (A.8), respectively.

Proof. See Appendix B.

Theorem 5. For the massive multiuser MIMO regime, the DL rate coverage probability of a typical MU associated with the $k$ th tier SBS is derived as

$$
\begin{aligned}
& \left.C_{k}^{\mathrm{DL}}\left(\mathrm{R}^{\mathrm{DL}}\right)\right|_{m \mathrm{M}} \\
= & \frac{2 \pi \lambda_{b^{k}}}{\Lambda_{k}^{\mathrm{DL}}} \int_{0}^{\infty} x\left[\frac{1}{2}-\frac{1}{\pi} \int_{0}^{\infty} \operatorname{Im}\left[\operatorname { e x p } \left\{\mathrm{jw} N_{o}\right.\right.\right. \\
& -\pi \lambda_{b^{\mathrm{M}}} \varpi_{1}(x, \mathrm{w})-\sum_{j=2}^{K} 2 \pi \lambda_{b^{j}}\left\{\varpi_{2}(x, \mathrm{w})\right. \\
& \left.+\varpi_{3}(x, \mathrm{w})\right\}-\pi \sum_{j=2}^{K} \lambda_{b^{j}}\left(\frac{P_{j} x^{\alpha_{k}}}{P_{k}}\right)^{\frac{2}{\alpha_{j}}}-\pi \lambda_{b^{\mathrm{M}}} \\
& \left.\left.\left.\left(\frac{P_{\mathrm{M}} \Psi}{P_{k}}\right)^{\frac{2}{\alpha_{\mathrm{M}}}} x^{\frac{2 \alpha_{k}}{\alpha_{\mathrm{M}}}}\right\}\left(1+\frac{\mathrm{jw} P_{k} \beta}{\gamma^{\mathrm{DL}} x^{\alpha_{k}}}\right)^{-1}\right]\right] \frac{d w}{\mathrm{w}} \mathrm{d} x,
\end{aligned}
$$

where

$$
\begin{aligned}
\varpi_{1}(x, \mathrm{w})= & \frac{\Gamma\left(1-\frac{2}{\alpha_{\mathrm{M}}}\right)+\frac{2}{\alpha_{\mathrm{M}}} \Gamma_{u}\left(-\frac{2}{\alpha_{\mathrm{M}}}, \frac{-\mathrm{jw} P_{\mathrm{M}} \beta}{\left(D_{k}^{\mathrm{M}}(x)\right)^{\alpha_{\mathrm{M}}}}\right)}{\left(-\mathrm{jw} P_{\mathrm{M}} \beta\right)^{\frac{2}{\alpha_{\mathrm{M}}}}} \\
& -\left(D_{k}^{\mathrm{M}}(x)\right)^{2}, \\
\varpi_{2}(x, \mathrm{w})= & \left(\frac{\left(\frac{P_{j}}{P_{k}}\right)^{2 / \alpha_{j}}(-\mathrm{jw}) \beta P_{k}\left(x^{\alpha_{k}}\right)^{2 / \alpha_{j}-1}}{\alpha_{j}-2}\right) \\
& { }_{2} F_{1}\left[1,1-\frac{2}{\alpha_{j}} ; 2-\frac{2}{\alpha_{j}} ; \frac{\mathrm{jw} \beta P_{j}}{x^{\alpha_{k}}}\left(D_{j}^{k}(x)\right)^{\alpha_{k}-\alpha_{j}}\right], \\
\varpi_{3}(x, \mathrm{w})= & \int_{0}^{\infty} \int_{0}^{r^{2}} \frac{1}{1+\left(-\mathrm{jw} \rho_{j} \beta^{(1-\epsilon)}\right)^{-1} u^{-\alpha_{j} \epsilon / 2} r^{\alpha_{j}}} \\
& \left(\pi \sum_{j=2}^{K} \lambda_{b^{j}} e^{-\pi \sum_{j=2}^{K} \lambda_{b^{j}} u}\right) \lambda_{I_{\Phi_{\mathrm{WU}}}}(r) \mathrm{d} u r \mathrm{~d} r
\end{aligned}
$$

and $\Lambda_{k}^{\mathrm{DL}}, \gamma^{\mathrm{DL}}, \Psi, D_{k}^{\mathrm{M}}(x)$, and $\lambda_{I_{\Phi} \mathrm{UL}}(r)$ are given in (19), (23), (18), (33), and (A.8), respectively.

Proof. The proof follows analogous steps to Theorem 4.

\section{Asymptotic UL Rate Coverage Probability}

We present the asymptotic UL rate coverage probability of a typical MU associated with the $k$ th tier SBS in Theorem 6.

Theorem 6. For the massive multiuser MIMO regime, the UL rate coverage probability of a typical MU associated with the $k$ th tier SBS is derived as

$$
\begin{aligned}
& \left.C_{k}^{\mathrm{UL}}\left(\mathrm{R}^{\mathrm{UL}}\right)\right|_{m \mathrm{M}} \\
& =\frac{2 \pi \lambda_{b^{k}}}{\Lambda_{k}^{\mathrm{UL}}} \int_{0}^{\infty} x\left[\frac{1}{2}-\frac{1}{\pi} \int_{0}^{\infty} \operatorname{Im}\left[\operatorname { e x p } \left\{\mathrm{jw} N_{o}-\pi \lambda_{b^{\mathrm{M}}}\right.\right.\right. \\
& \vartheta_{1}(x, \mathrm{w})-\sum_{j=2}^{K} 2 \pi \lambda_{b^{j}}\left\{\vartheta_{2}(x, \mathrm{w})+\vartheta_{3}(x, \mathrm{w})\right\}-\sum_{j=2}^{K} \pi \lambda_{b^{j}} x^{\frac{2 \alpha_{k}}{\alpha_{j}}} \\
& \left.\left.\left.\left(\frac{P_{j}}{P_{k}}\right)^{\frac{2}{\alpha_{j}}}\right\}\left(1+\frac{\mathrm{jw} \rho_{k} x^{\alpha_{k} \epsilon} \beta^{-\epsilon} \beta}{\gamma^{U L} x^{\alpha_{k}}}\right)^{-1}\right]\right] \frac{\mathrm{dw}}{\mathrm{w}} d x
\end{aligned}
$$

where

$$
\begin{aligned}
\vartheta_{1}(x, \mathrm{w})= & \left(-\mathrm{jw} P_{\mathrm{M}} \beta\right)^{\frac{2}{\alpha_{\mathrm{M}}}} \Gamma\left(1-\frac{2}{\alpha_{\mathrm{M}}}\right), \\
\vartheta_{2}(x, \mathrm{w})= & \left(-\mathrm{jw} P_{j} \beta\right) \frac{x^{2-\alpha_{j}}}{\left(\alpha_{j}-2\right)} \\
& { }_{2} F_{1}\left[1,1-\frac{2}{\alpha_{j}} ; 2-\frac{2}{\alpha_{j}} ; \frac{\mathrm{jw} \beta P_{j}}{x^{\alpha_{k}}} x^{\left(\alpha_{k}-\alpha_{j}\right)}\right], \\
\vartheta_{3}(x, \mathrm{w})= & \int_{0}^{\infty} \int_{0}^{r^{2}} \frac{1}{1+\left(-\mathrm{jw} \rho_{j} \beta^{1-\epsilon}\right)^{-1} u^{-\alpha_{j} \epsilon / 2} r^{\alpha_{j}}} \\
& \left(\pi \sum_{j=2}^{K} \lambda_{b^{j}} e^{-\pi \sum_{j=2}^{K} \lambda_{b^{j}} u}\right) \mathrm{d} u r \mathrm{~d} r,
\end{aligned}
$$

and $\Lambda_{k}^{\mathrm{UL}}$ and $\gamma^{\mathrm{UL}}$ are given in (20) and (38), respectively.

Proof. The proof follows analogous steps to Theorem 4. 
TABLE II: Parameter Values unless specified

\begin{tabular}{cc|cc}
\hline Parameter & Value & Parameter & Value \\
\hline \hline$\lambda_{b^{\mathrm{M}}}$ & $5 \times 10^{-5} / \mathrm{m}^{2}$ & $\mu$ & 20 \\
\hline$P_{\mathrm{M}}$ & $40 \mathrm{dBm}$ & $P_{2}$ & $33 \mathrm{dBm}$ \\
\hline$\alpha_{\mathrm{M}}$ & 3.5 & $\alpha_{2}$ & 4 \\
\hline$N$ & 128 & $U_{M}$ & 5 \\
\hline$\rho_{2}$ & $-40 \mathrm{dBm}$ & $\epsilon$ & 0.9 \\
\hline $\mathrm{R}^{\mathrm{DL}}$ & 0.5 nats/sec/Hz & $\mathrm{R}^{\mathrm{UL}}$ & 0.5 nats $/ \mathrm{sec} / \mathrm{Hz}$ \\
\hline$N_{0}$ & $-100 \mathrm{dBm}$ & $P_{k} h_{L I}$ & 0 \\
\hline
\end{tabular}

Even though the asymptotic DL and UL rate coverage probabilities' expressions in Theorem 4, 5, and 6 are composed of four integrals, they can be computed in less time compared to the corresponding simulations.

\section{Performance Comparison with the CONVENTIONAL HD HCNS}

In order to compare the performance of the proposed $\mathrm{HCNs}$ with FD SBSs with that of the conventional HD HCNs with HD SBSs, we define the total ASE of a random MU in HCNs as

$$
\mathrm{ASE}=\mathrm{ASE}^{\mathrm{DL}}+\mathrm{ASE}^{\mathrm{UL}},
$$

where $\mathrm{ASE}^{\mathrm{DL}}$ and $\mathrm{ASE}^{\mathrm{UL}}$ are given in (35) and (41), respectively. Furthermore, we define the ASE of FD small cell MUs as

$$
\mathrm{ASE}_{\mathrm{SBS}}=\mathrm{ASE}_{\mathrm{SBS}}^{\mathrm{DL}}+\mathrm{ASE}^{\mathrm{UL}}
$$

where $\mathrm{ASE}_{\mathrm{SBS}}^{\mathrm{DL}}$ and $\mathrm{ASE}^{\mathrm{UL}}$ are given in (35) and (41), respectively.

\section{NumericAl Results}

In this section, we investigate the system performance in the DL and the UL in terms of the rate coverage probability and the ASE of HCNs with multiuser MIMO antennas at the MBSs and FD operation at the SBSs. We compare the performance of HCNs with multiuser MIMO at MBSs and FD at SBSs with that of massive multiuser MIMO at MBSs and FD at SBSs. We plot the DL rate coverage probability, the DL ASE, the UL rate coverage probability, and the UL ASE using (21), (35), (36), and (41), respectively. We validate the accuracy of the derived expressions for a two-tier HCNs with network radius $A_{n}=\pi(1000)^{2} \mathrm{~km}^{2}$ consisting of HD macrocells with density $\lambda_{b^{\mathrm{M}}}$ and FD small cells with density $\lambda_{b^{2}}$, via Monte Carlo simulations. The interference approximations in Section II-H are not made in the simulation. The simulation is repeated and averaged over 10,000 iterations. The results presented in the figures of this section validate the accuracy of our approach to characterize the interferences and show that the assumptions made have a minor effect on the accuracy of the proposed analytical model. Unless specified, the parameter values used in this section are listed in Table II.

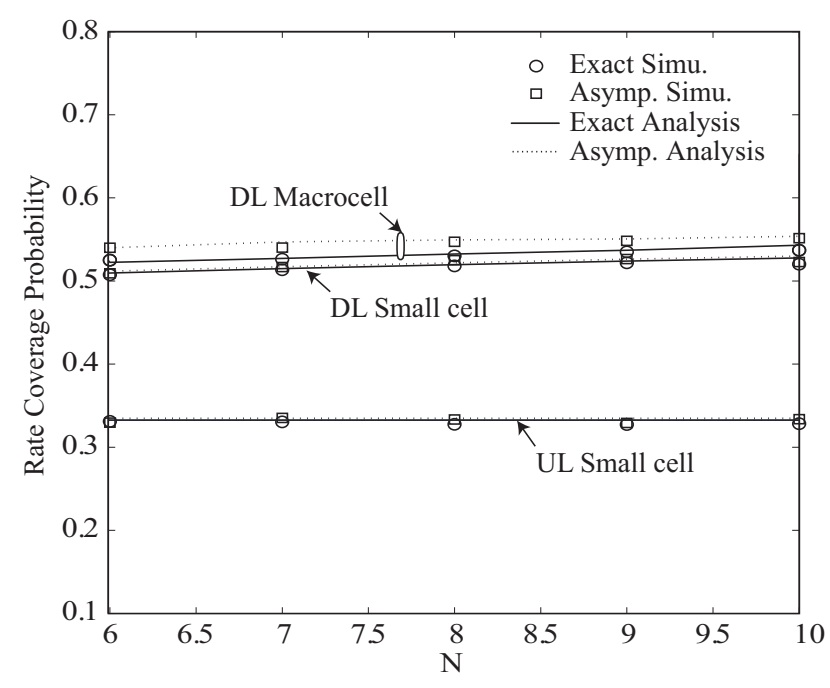

(a) Rate Coverage Probability versus small $N$ (multiuser MIMO).

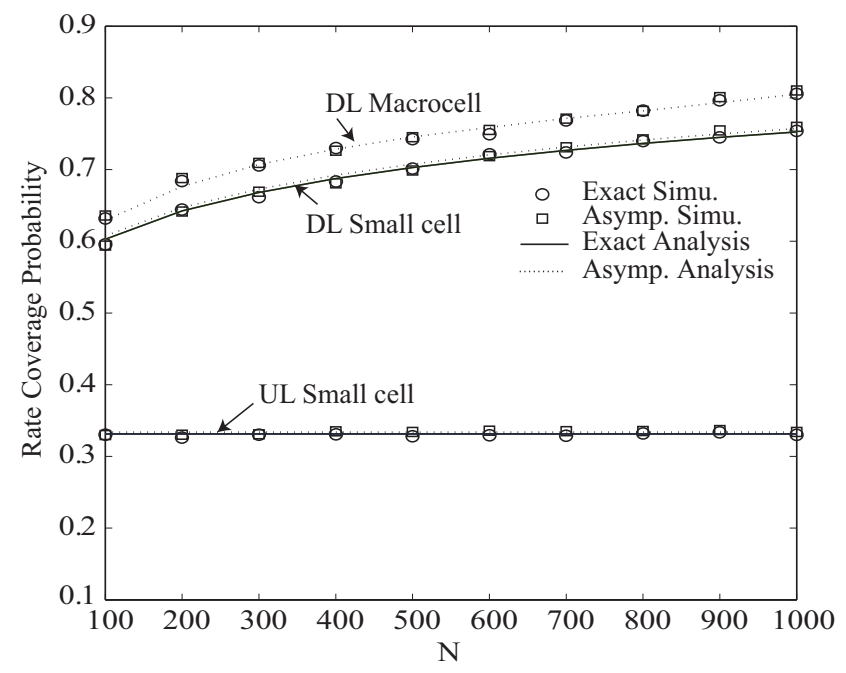

(b) Rate Coverage Probability versus large $N$ (massive multiuser MIMO)

Fig. 2: Rate coverage probability versus the number of MBS antennas.

\section{A. Impact of number of multiuser MIMO/massive multiuser MIMO antennas at the MBS on the DL and UL Rate Coverage Probability}

Fig. 5(a) and Fig. 5(b) compare the DL and the UL rate coverage probability with the multiuser MIMO at the MBS to that with massive multiuser MIMO at the MBS as a function of the number of antennas at the MBS. We see that the asymptotic rate coverage probability of small cell MU closely matches the exact rate coverage probability in DL and UL both for small and large $N$. This observation can be attributed to the fact that changing $N$ at the interfering MBS does not change the distributions of short term fading factors $h_{x, \mathrm{u}_{0}^{k}}$ in (10) and $h_{x, b_{0}^{k}}$ in (14), for the exact case, which are ignored in the asymptotic case due to channel hardening effect. As expected, the DL rate coverage probabilities of the macrocell $\mathrm{MU}$ and the small cell MU in massive multiuser MIMO case is higher 
than those in multiuser MIMO case due to the large antenna array gain. However, the UL rate coverage probability of MU remains constant with increasing $N$ for both small and large $N$ due to that: 1) the UL MU can only associate with the SBSs, and 2) the interferences from $N$ MBS antennas do not add coherently such that for the same total transmit power, the interference level from a MBS to an UL MU is the same, regardless of the number of $N$ under i.i.d. Rayleigh fading channels.

\section{B. Impact of number of SBSs density on the DL and ULASE}

Fig. 3 compares the DL and the UL rate coverage probability with massive multiuser MIMO at the MBS as a function of the ratio between the SBSs density to the MBSs density $\left(\mu=\lambda_{b^{2}} / \lambda_{b^{\mathrm{M}}}\right)$. The increase in $\lambda_{b^{2}}$ improves the DL rate coverage probabilities of macrocell $\mathrm{MU}$ and small cell $\mathrm{MU}$. This is according to the the fact that increasing $\lambda_{b^{2}}$ decreases the distance between the typical small cell MU and the serving SBS. Thus, the MUs transmit with less power due to distanceproportional fractional power control, which in turn reduces the UL interference for the macrocell MU and the small cell MU. However, increasing $\lambda_{b^{2}}$ decreases the UL rate coverage probability due to the increased interference from larger number of SBSs.

\section{Performance comparison of the proposed HCNs with the conventional HCNs}

Fig. 4 compares the ASE of the proposed HCNs with FD SBSs to that of the conventional HCNs with HD SBSs. We plot the ASE of a random MU in the proposed HCNs using (60), and that in the conventional HCNs using $\mathrm{ASE}^{\mathrm{DL}}$ in (35) with no UL interference from the MUs, i.e., $I_{u_{u l}^{s}}, u_{0}^{\mathrm{M}}=0$ in (5) and $I_{u_{u l}^{s}, u_{0}^{k}}=0$ in (9). We plot the ASE of a small cell MU of the proposed HCNs using (61), and that of conventional HCNs using ASE $\mathrm{SBS}_{\mathrm{SB}}^{\mathrm{DL}}$ in (35) with no UL interference from the MUs, i.e., $I_{u_{u l}^{s}, u_{0}^{k}}=0$ in (9). The ASE of the proposed HCNs is observed to be higher than that of the conventional HCNs. This suggests the ASE improvement brought by simultaneous transmission in DL and UL due to FD SBSs which dominates the resulting additional interferences. With the increase in the number of antennas at the MBSs, the ASE of the HCNs increases due to the increase in the rate coverage probability with larger $N$ as shown in Fig. 2b. Moreover, similar trends are observed for the small cell tier with improved ASE than that of the HCNs.

\section{Impact of SBS density with different number of MBS antennas on the DL Performance}

Fig. 5(a) and Fig. 5(b) examine the trade-off between the DL ASE and the DL rate coverage probability versus the ratio between density of SBSs to density of MBS $\left(\mu=\lambda_{b^{2}} / \lambda_{b^{M}}\right)$ and the number of MBS antennas both for multiuser MIMO and massive multiuser MIMO at the MBSs. In Fig. 5(a) and Fig. 5(b), we consider $\epsilon=0$ and the transmit power at the MU $P_{u}$ is taken as $23 \mathrm{dBm}$. Clearly, the DL ASE and rate coverage probability with massive multiuser MIMO at the

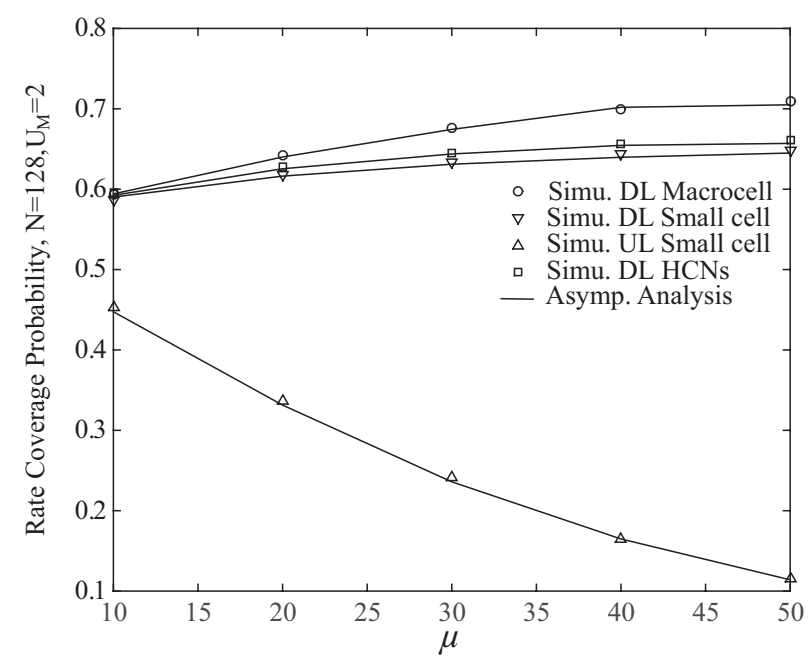

Fig. 3: Rate coverage probability versus the ratio between SBSs density to MBSs density.

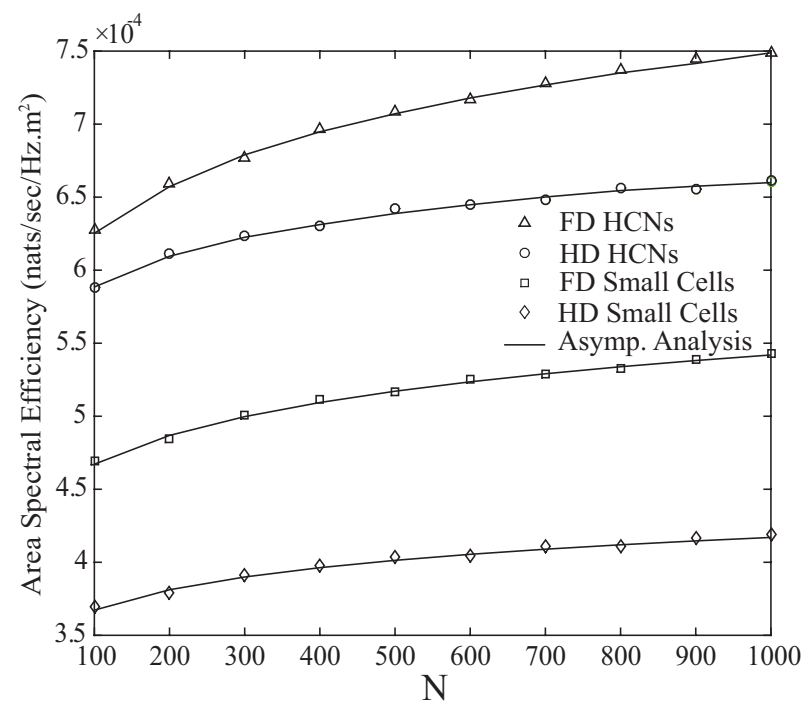

Fig. 4: ASE versus the number of MBS antennas.

MBS are higher as compared to those with multiuser MIMO at the MBSs due to sharp beamforming. The massive number of antennas at MBSs brings the higher DL rate coverage probability and ASE.

The DL ASE and the rate coverage probability of macrocell MU decreases with increasing the SBSs density due to the increased interference from SBSs. However, increasing the SBSs density $\lambda_{b^{k}}$ increases the DL ASE, but decreases the DL rate coverage probability of small cell MU. With the increase in the SBSs density, the number of DL small cell transmissions and the aggregate interference from small cells increase, which results in a trade-off between DL ASE and rate coverage probability for the small cell MUs. We have shown that the UL interference can be reduced by employing UL power control in the UL, which improves the DL rate coverage probability in Fig. 3. 


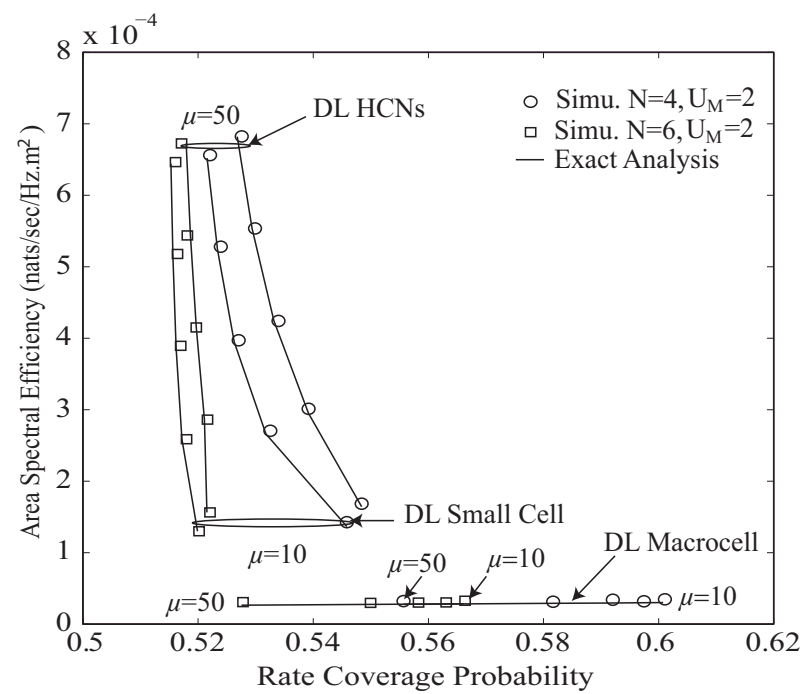

(a) ASE versus Rate Coverage Probability, small $N$ (multiuser MIMO).

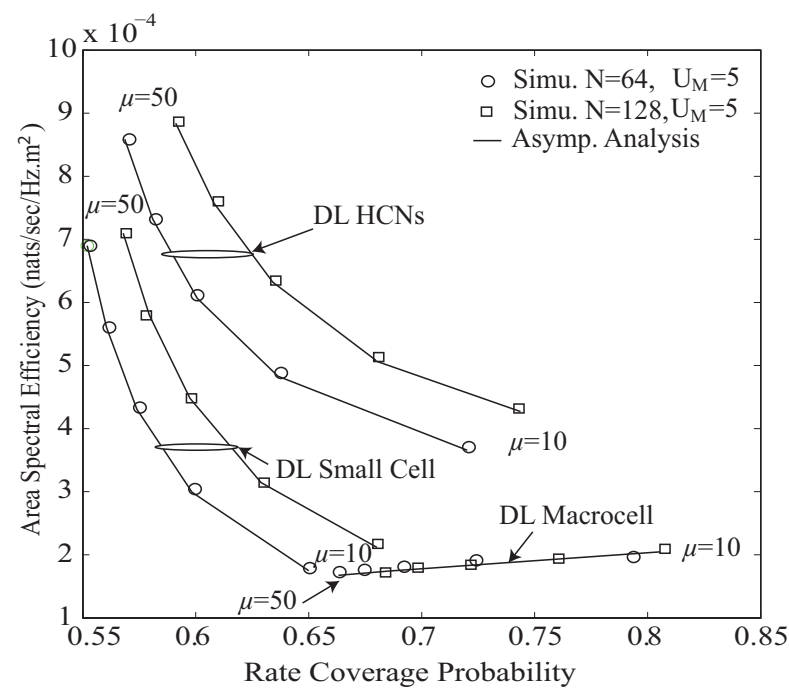

(b) ASE versus Rate Coverage Probability, large $N$ (massive multiuser MIMO).

Fig. 5: The tradeoff between the ASE and the rate coverage probability for various number of MBS antennas.

\section{E. Impact of SBS density with different MBS and SBS transmit powers on the DL and UL Performance}

Fig. 6 plots the DL and UL ASE and rate coverage probability as a function of the transmit powers at the MBSs and SBSs. In Fig. 6, we consider $\epsilon=0$ and the transmit power at the MU $P_{u}$ is taken as $23 \mathrm{dBm}$. Increasing the MBS transmit power increases the DL ASE and the rate coverage probability of all tiers, which is due to the increase of $\operatorname{SINR}_{\mathrm{M}}^{\mathrm{DL}}$ in (5), and the reduced distance between the typical small cell MU and the associated SBS. Moreover, we observe the decrease in the UL ASE and the UL rate coverage probability with the increase in $P_{\mathrm{M}}$ and $P_{k}$, which is due to the increased cross-tier and co-tier interferences as can be seen from (13). Furthermore, we observe that the increase in the SBS density increases the UL rate coverage probability in contrast to the decreased DL rate coverage probability for small cell MU as

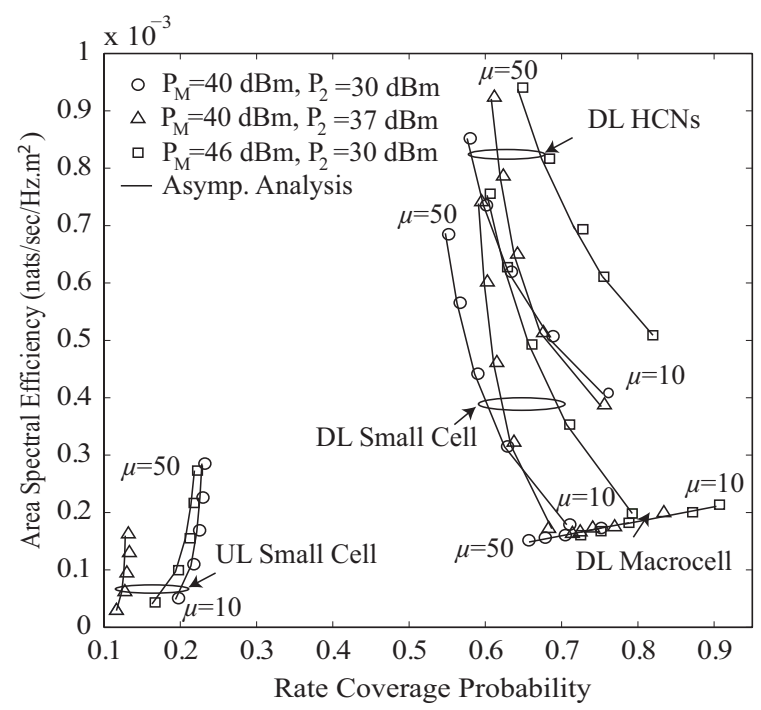

Fig. 6: The tradeoff between the ASE and the rate coverage probability for various MBS and SBS transmit powers.

shown in Fig. 5a and Fig. 5b, which is due to the decreased distance between the UL small cell MU and the serving SBS. It can thus be concluded that the SBS density and the BS transmit power of each tier can be tuned to achieve joint DL and UL performance gains with FD SBSs.

\section{F. Impact of SI cancellation capability with different SBS transmit power on the DL and UL Performance}

Fig. 7 examines the impact of the SI cancellation capability $L_{d B}$ on the DL and UL rate coverage probabilities. As expected, increasing $L_{d B}$ decreases the UL rate coverage probability of the small cell MU. Moreover, increasing the SBS transmit power decreases the UL rate coverage probability of the small cell MU, due to the increased self interference. However, increasing the SBS transmit power increases the DL rate coverage probability of a random $\mathrm{MU}$, due to the increase of $\mathrm{SINR}_{\mathrm{k}}^{\mathrm{DL}}$ in (44).

\section{G. Impact of receiver sensitivity at the SBS with different power control factors}

Fig. 8 plots the DL and the UL rate coverage probability versus the receiver's sensitivity at SBSs $\rho_{2}$ for various power control factors $\epsilon$. Increasing $\rho_{2}$ increases the UL rate coverage probability, and degrades the DL rate coverage probability. This is due to the reason that decreasing the the SBS receiver sensitivity (i.e., an increase in $\rho_{2}$ ) increases the transmit power required at each MU to perform channel inversion towards serving SBS, which in turn increases the useful signal power at the its associated SBS and the interference at the other BSs and MUs. Similarly, higher power control factor $\epsilon$ improves the UL performance, but degrades the DL performance. These results demonstrate that $\rho_{2}$ and $\epsilon$ can be optimized for joint DL and UL performance gain. We also compare the DL and UL performance of HCNs with UL power control to that without UL power control when the MUs transmit power is $P_{u}=23$ 


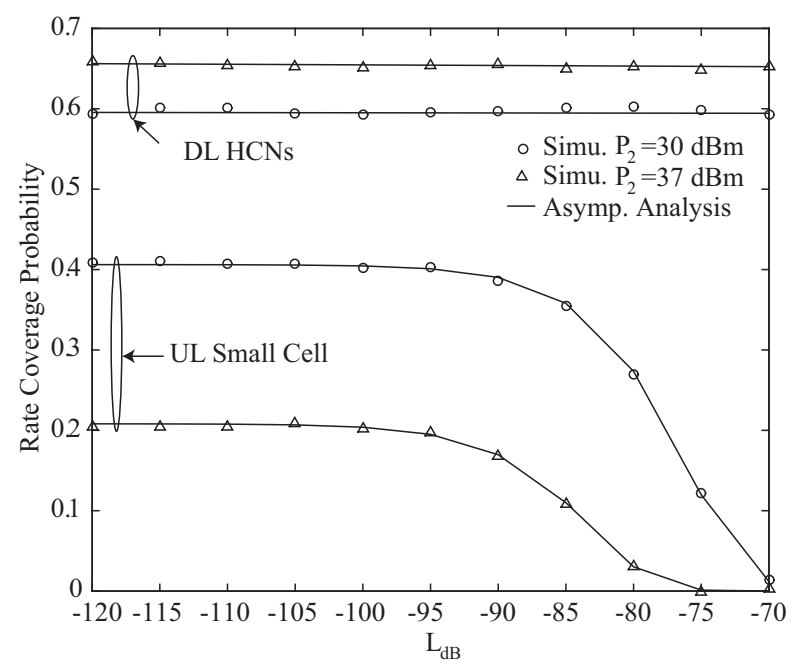

Fig. 7: Rate coverage probability versus SI cancellation capability for various SBSs transmit powers.

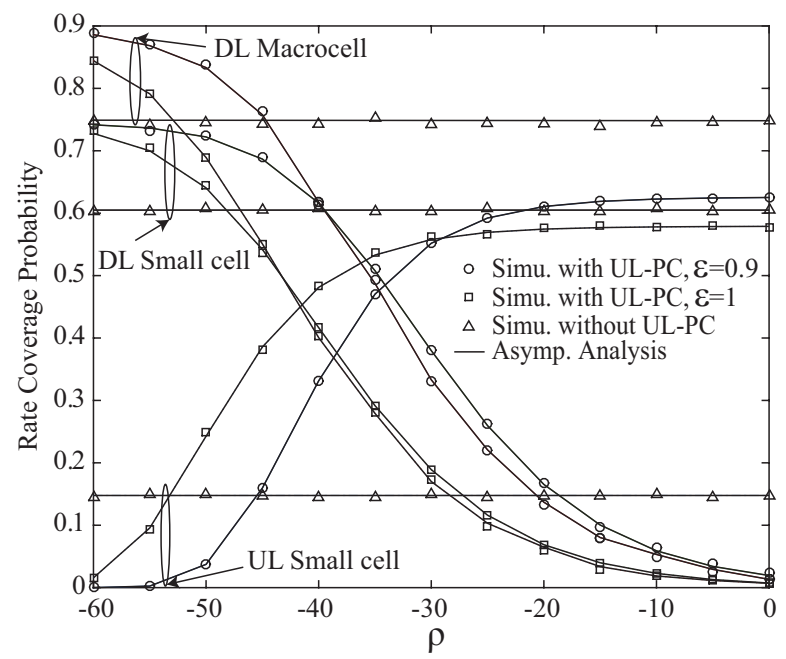

Fig. 8: Rate coverage probability versus SBSs receivers sensitivity for various SBSs power control factors.

$\mathrm{dBm}$. The UL rate coverage probability in HCNs without UL power control is shown to be very small due to the increased inter-cell interference from the UL MUs.

\section{CONCLUSION}

In this paper, we have presented a tractable model for massive multiuser MIMO-enabled HCNs with FD small cells. Relying on stochastic geometry, we have derived the analytical expressions for DL rate coverage probability and ASE for macrocell and small cells, and UL rate coverage probability and ASE for small cells. We have also presented the asymptotic expressions as the number of antennas at MBS goes to infinity. Numerical results demonstrated the benefits brought by massive multiuser MIMO in achieving high rate coverage probability and the benefits brought by of FD SBSs in achieving high ASE. It is shown that the SBSs density and the number of antennas at the MBSs can be used as design parameters to target optimal DL ASE and DL rate coverage probability. The results also demonstrate that, to achieve similar performance in the DL and the UL, UL power control should be employed. With the advancements of massive multiuser MIMO and SI cancellation in FD, the proposed HCNs will prove to be a promising candidate for $5 \mathrm{G}$ systems.

\section{APPENDIX A}

ProOF OF THEOREM 1

From (22), the rate coverage probability of the macrocell tier is given by deconditioning over $\left|X_{o, u_{0}^{\mathrm{M}}}\right|$ as

$C_{\mathrm{M}}=\int_{0}^{\infty} \operatorname{Pr}\left[\left.\operatorname{SINR}_{\mathrm{M}}^{\mathrm{DL}}\right|_{\left|X_{o, u_{0}^{\mathrm{M}}}\right|=x} \geq \gamma^{\mathrm{DL}}|| X_{o, u_{0}^{\mathrm{M}}} \mid\right] f_{\left|X_{o, \mathrm{M}}\right|}(x) \mathrm{d} x$

where $f_{\left|X_{o, \mathrm{M}}\right|}(x)$ is the PDF of the distance between a typical MU and its serving MBS given by [44] as follows

$$
\begin{aligned}
f_{\left|X_{o, \mathrm{M}}\right|}(x)= & \frac{2 \pi \lambda_{b^{\mathrm{M}}}}{\Lambda_{\mathrm{M}}} x \exp \left\{-\pi \lambda_{b^{\mathrm{M}}} x^{2}-\pi \sum_{j=2}^{K} \lambda_{b^{j}}\right. \\
& \left.\left(\frac{P_{j}}{\Psi P_{\mathrm{M}}}\right)^{2 / \alpha_{j}} x^{2 \alpha_{\mathrm{M}} / \alpha_{j}}\right\},
\end{aligned}
$$

Substituting $\operatorname{SINR}_{\mathrm{M}}^{\mathrm{DL}}(\mathrm{x})$ from (5) into (A.1) and simplifying we obtain

$$
\begin{aligned}
& \operatorname{Pr}\left(\frac{\frac{P_{\mathrm{M}}}{U_{\mathrm{M}}} \beta g_{o, u_{0} \mathrm{M}} x^{-\alpha_{\mathrm{M}}}}{I_{u_{0}^{\mathrm{M}}}+N_{0}}>\tau\right) \\
& \stackrel{(a)}{=} \int_{0}^{\infty} e^{-\frac{\tau U_{\mathrm{M}} x^{\alpha_{\mathrm{M}}\left(\gamma^{\mathrm{DL}}+N_{0}\right)}}{P_{\mathrm{M}} \beta}} \sum_{n=0}^{N-U_{\mathrm{M}}} \frac{\left(\frac{\tau U_{\mathrm{M}} x^{\alpha_{\mathrm{M}}}\left(\gamma^{\mathrm{DL}}+N_{0}\right)}{P_{\mathrm{M}} \beta}\right)^{n}}{n !} \\
& \quad d \operatorname{Pr}\left(I_{u_{0}^{M}} \leq \gamma^{\mathrm{DL}}\right), \\
& \left.\stackrel{(b)}{=} \sum_{n=0}^{N-U_{\mathrm{M}}} \frac{\left(x^{\alpha_{\mathrm{M}}}\right)^{n}}{n !(-1) !} \frac{d^{n}\left(e^{-\frac{\tau U_{\mathrm{M}} x^{\alpha_{\mathrm{M}} N_{0}}}{P_{\mathrm{M}} \beta}} \mathcal{L}_{I_{u_{0}^{\mathrm{M}}}}\left(\frac{\tau U_{\mathrm{M}} q}{P_{\mathrm{M}} \beta}\right)\right)}{d q^{n}}\right|_{q=x^{\alpha_{\mathrm{M}}}}
\end{aligned}
$$

where (a) follows from $g_{o, \mathrm{u}_{0}^{\mathrm{M}}} \sim \Gamma\left(N-U_{\mathrm{M}}+1,1\right)$ and (b) follows from some some mathematical manipulations. In (A.3), $\mathcal{L}_{I_{u_{0}^{\mathrm{M}}}}$ is the Laplace transform of the PDF of $I_{u_{0}^{M}}$ given as

$$
\mathcal{L}_{I_{u_{0}^{\mathrm{M}}}}(s)=\mathcal{L}_{I_{\mathrm{M}, u_{0}^{\mathrm{M}}}}(s) \mathcal{L}_{I_{S, u_{0}^{\mathrm{M}}}}(s) \mathcal{L}_{I_{u}^{S}, u_{0}^{\mathrm{M}}}(s),
$$

where $\mathcal{L}_{I_{\mathrm{M}, u_{0}^{\mathrm{M}}}}(s), \mathcal{L}_{I_{S, u_{0}^{\mathrm{M}}}}(s)$, and $\mathcal{L}_{I_{u} S}, u_{0}^{\mathrm{M}}(s)$ are the Laplace transform of the PDF of $I_{\mathrm{M}, u_{0}^{\mathrm{M}}}, I_{S, u_{0}^{\mathrm{M}}}$, and $I_{u_{u l}^{S}, u_{0}^{\mathrm{M}}}$, respectively. In (A.4), $\mathcal{L}_{I_{\mathrm{M}, u_{0}^{\mathrm{M}}}}(s)$ is derived as

$$
\begin{aligned}
& \mathcal{L}_{I_{\mathrm{M}, u_{0}^{\mathrm{M}}}}(s) \\
& =\mathbb{E}\left[\exp \left\{-s \sum_{m \in \Phi_{b}^{\mathrm{M}} \backslash 0} \frac{P_{\mathrm{M}}}{U_{\mathrm{M}}} h_{m, u_{0}^{\mathrm{M}}} \beta\left|X_{m, u_{0}^{\mathrm{M}}}\right|^{-\alpha_{\mathrm{M}}}\right\}\right] \\
& \stackrel{(a)}{=} \exp \left\{-2 \pi \lambda_{b^{\mathrm{M}}} \sum_{\nu=1}^{U_{\mathrm{M}}}\left(\begin{array}{c}
U_{\mathrm{M}} \\
\nu
\end{array}\right) \int_{x}^{\infty} \frac{\left(\frac{P_{\mathrm{M}}}{U_{\mathrm{M}}} \beta\right)^{\nu} s^{\nu}\left(r^{-\alpha_{\mathrm{M}}}\right)^{\nu}}{\left(1+s \frac{P_{\mathrm{M}}}{U_{\mathrm{M}}} \beta r^{-\alpha_{\mathrm{M}}}\right)^{U_{\mathrm{M}}}} r \mathrm{~d} r\right\}
\end{aligned}
$$


where (a) is obtained by using generating functional of PPP [48], $h_{m, u_{0}^{\mathrm{M}}} \sim \Gamma\left(U_{\mathrm{M}}, 1\right)$, and using Binomial expansion. Likewise, $\mathcal{L}_{I_{S, u_{0}^{\mathrm{M}}}}(s)$ is evaluated as

$$
\begin{gathered}
\mathcal{L}_{I_{S, u_{0}^{\mathrm{M}}}}(s)=\exp \left\{-\sum_{j=2}^{K} 2 \pi \lambda_{b^{j}} \int_{D_{j}^{\mathrm{M}}(x)}^{\infty}\left(\frac{s P_{j} \beta r^{-\alpha_{j}}}{1+s P_{j} \beta r^{-\alpha_{j}}}\right)\right. \\
r \mathrm{~d} r\},
\end{gathered}
$$

where $D_{j}^{\mathrm{M}}(x)$ is the distance between a typical MU and the closest interfering BS in the $j$ th tier given in (30). In (A.4), $\mathcal{L}_{I_{u l}^{S}, u_{0}^{\mathrm{M}}}(s)$ is evaluated as

$$
\begin{gathered}
\mathcal{L}_{I_{u_{u l}^{s}, u_{0}^{\mathrm{M}}}}(s) \stackrel{(a)}{=} \exp \left\{-\sum_{j=2}^{K} 2 \pi \lambda_{b^{j}} \int_{0}^{\infty}\left(1-\exp \left(-\pi \frac{\lambda_{b^{j}}}{A_{j}^{U L}} r^{2}\right)\right)\right. \\
\left.\mathbb{E}_{R}\left[\frac{s \rho_{j} \beta^{1-\epsilon} R^{\epsilon \alpha_{j}} r^{-\alpha_{j}}}{1+s \rho_{j} \beta^{1-\epsilon} R^{\epsilon \alpha_{j}} r^{-\alpha_{j}}}\right] r d r\right\},
\end{gathered}
$$

where (a) follows from the probability generating functional of a PPP and the fact that the UL interference field is a nonhomogeneous PPP with distance dependent density function given as

$$
\lambda_{I_{\Phi_{b j}}(r)}(r) \lambda_{b^{j}}\left(1-\exp \left(-\pi \frac{\lambda_{b^{j}}}{A_{j}^{U L}} r^{2}\right)\right)
$$

where $\left(A_{j}^{U L}=\lambda_{b^{j}} / \sum_{i=2}^{K} \lambda_{b^{i}}\right)$ is the repulsion parameter as in [49]. In (A.8), the integral has a lower limit of zero as the nearest UL MU of FD SBS can be arbitrarily close to the typical macrocell MU. Using the PDF of serving link distances given in (C.1), we derive $\mathcal{L}_{I_{u_{u l}^{s}}, u_{0}^{\mathrm{M}}}(s)$. Plugging (A.5), (A.6) and (A.7) into (A.4), after some manipulations, $\mathcal{L}_{I_{u_{0}^{\mathrm{M}}}}(s)$ is derived as

$$
\mathcal{L}_{I_{u_{0}^{\mathrm{M}}}}(s)=e^{-\zeta(s)},
$$

where $\zeta(s)$ is given by (27). Substituting (A.9) into (A.3), simplifying using the Faa di Bruno's formula, and finally plugging into (A.1), we obtain (25).

\section{APPENDIX B \\ PROOF OF THEOREM 2}

The rate coverage probability of a typical MU associated with the $k$ th tier SBS is evaluated following the similar steps as of Theorem 1 with the PDF of the distance between a typical DL MU and its serving SBS is given by [44]

$$
\begin{aligned}
\left.f_{\left|X_{o, k}\right|}(x)\right|_{D L}= & 2 \pi \lambda_{b^{k}} r \exp \left\{-\pi \sum_{j=2}^{K} \lambda_{b^{j}}\left(P_{j} r^{\alpha_{\mathrm{k}}} / P_{k}\right)^{2 / \alpha_{j}}\right. \\
& \left.-\pi \lambda_{b^{\mathrm{M}}}\left(\frac{P_{\mathrm{M}} \Psi}{P_{k}}\right)^{2 / \alpha_{M}} r^{2 \alpha_{k} / \alpha_{\mathrm{M}}}\right\}
\end{aligned}
$$

where $\Psi$ is given in (18).

\section{APPENDIX C \\ Proof OF THEOREM 3}

The UL rate coverage probability of a typical MU associated with the $k$ th tier SBS is evaluated following the similar steps as of Theorem 1 with the PDF of the distance between a typical UL MU and its serving SBS is given by [44]

$$
\left.f_{\left|X_{o, k}\right|}(x)\right|_{U L}=2 \pi \lambda_{b^{k}} r \exp \left\{-\sum_{j=2}^{K} \pi \lambda_{b^{j}} r^{2 \alpha_{k} / \alpha_{j}}\right\} .
$$

\section{APPENDIX D \\ PROOF OF THEOREM 4}

Based on (42), the DL rate coverage probability of the macrocell with massive multiuser MIMO at the BSs, can be given as

$\left.C_{\mathrm{M}}\right|_{m \mathrm{M}}=\int_{0}^{\infty} F_{\left.I_{u_{0}^{\mathrm{M}}}\right|_{m \mathrm{M}}}\left(\frac{P_{\mathrm{M}} \beta}{\gamma_{\mathrm{M}_{m \mathrm{M}}}^{\mathrm{DL}}\left|X_{o, u_{0}^{\mathrm{M}}}\right|^{\alpha_{\mathrm{M}}}}-N_{0}\right) f_{\left|X_{o, \mathrm{M}}\right|}(x) \mathrm{d} x$,

where we resort to apply the Gil-Pelaez inversion theorem [50] and the CDF of the interference $\left.F_{I_{u_{0}^{\mathrm{M}}}}\right|_{m \mathrm{M}}$ (.) can be derived as

$$
\begin{aligned}
& F_{\left.I_{u_{0}^{\mathrm{M}}}\right|_{m \mathrm{M}}}(x) \\
& =\frac{1}{2}-\frac{1}{\pi} \int_{0}^{\infty} \operatorname{Im}\left[\frac{\mathcal{L}_{I_{\mathrm{M}_{m \mathrm{M}}, u_{0}^{\mathrm{M}}}}(-\mathrm{jw}) \mathcal{L}_{I_{S, u_{0}^{\mathrm{M}}}}(-\mathrm{jw}) \mathcal{L}_{u_{u l}^{s}, u_{0}^{\mathrm{M}}}(-\mathrm{jw})}{\exp \left(\mathrm{jw}\left(\frac{P_{\mathrm{M}} \beta}{\gamma_{\mathrm{M}_{m \mathrm{M}}^{\mathrm{D}}}^{\mathrm{D}} x^{\alpha_{\mathrm{M}}}}-N_{0}\right)\right)}\right] \frac{\mathrm{dw}}{\mathrm{w}},
\end{aligned}
$$

where $\operatorname{Im}($.$) represents the imaginary part of the argument. In$ (D.2), the Laplace transform of $I_{\mathrm{M}_{m \mathrm{M}}, u_{0}^{k}}$ can be derived as under

$$
\begin{aligned}
& \mathcal{L}_{I_{\mathrm{M}_{m \mathrm{M}}, u_{0}^{\mathrm{M}}}}(-\mathrm{jw}) \\
& \stackrel{(a)}{=} \exp \left\{-2 \pi \lambda_{b^{\mathrm{M}}} \int_{x}^{\infty}\left(1-\exp \left\{-(-\mathrm{jw}) P_{\mathrm{M}} \beta r^{\alpha_{\mathrm{M}}}\right\}\right) r \mathrm{~d} r\right\},
\end{aligned}
$$

where (a) follows from the probability generating functional of PPP. Solving the integral in (D.3) we derive $\mathcal{L}_{I_{\mathrm{M}_{m \mathrm{M}}, u_{0}^{k}}}(-\mathrm{jw})$.

In (D.2), the Laplace transforms of $I_{S, u_{0}^{\mathrm{M}}}$ and $I_{u_{u l}^{s}}^{s}, u_{0}^{\mathrm{M}}$ can be evaluated as (A.6) and (A.7), respectively. Finally substituting $\mathcal{L}_{I_{\mathrm{M}_{m \mathrm{M}}}, u_{0}^{\mathrm{M}}}(-\mathrm{jw}), \mathcal{L}_{I_{S, u_{0}^{\mathrm{M}}}}(-\mathrm{jw})$, and $\mathcal{L}_{I_{u_{u}}, u_{0}^{\mathrm{M}}}(-\mathrm{jw})$ into (D.2), and plugging (D.2) into (D.1), we obtain Theorem 4.

\section{REFERENCES}

[1] J. Andrews, S. Buzzi, W. Choi, S. Hanly, A. Lozano, A. Soong, and J. Zhang, "What will 5G be?" IEEE J. Sel. Areas Commun., vol. 32, no. 6, pp. 1065-1082, Jun. 2014.

[2] S. Parkvall, A. Furuskar, and E. Dahlman, "Evolution of LTE towards IMT-advanced," IEEE Commun. Mag., vol. 49, no. 2, pp. 84-91, Feb. 2011.

[3] H. Q. Ngo, E. G. Larsson, and T. L. Marzetta, "Energy and spectral efficiency of very large multiuser MIMO systems," IEEE Trans. Commun., vol. 61, no. 4, pp. 1436-1449, Apr. 2013.

[4] A. Sabharwal, P. Schniter, D. Guo, D. W. Bliss, S. Rangarajan, and R. Wichman, "In-band full-duplex wireless: Challenges and opportunities," IEEE J. Sel. Areas Commun., vol. 32, no. 9, pp. 1637-1652, Sept. 2014. 
[5] T. Snow, C. Fulton, and W. J. Chappell, "Transmit-receive duplexing using digital beamforming system to cancel self-interference," IEEE Trans. Microw. Theory Tech., vol. 59, no. 12, pp. 3494-3503, Dec. 2011.

[6] T. Riihonen, S. Werner, and R. Wichman, "Mitigation of loopback selfinterference in full-duplex MIMO relays," IEEE Trans. Signal Process. vol. 59, no. 12, pp. 5983-5993, Dec. 2011.

[7] T. Riihonen, S. Werner, and R. Wichman, "Hybrid full-duplex/halfduplex relaying with transmit power adaptation," IEEE Trans. Wireless Commun., vol. 10, no. 9, pp. 3074-3085, Sep. 2011.

[8] G. Liu, F. R. Yu, H. Ji, V. C. M. Leung, and X. Li, "In-band full-duplex relaying: A survey, research issues and challenges," IEEE Communications Surveys Tutorials, vol. 17, no. 2, pp. 500-524, Secondquarter 2015.

[9] H. Ju, E. Oh, and D. Hong, "Improving efficiency of resource usage in two-hop full duplex relay systems based on resource sharing and interference cancellation," IEEE Trans. Wireless Commun., vol. 8, no. 8 , pp. 3933-3938, Aug. 2009.

[10] D. Nguyen, L. N. Tran, P. Pirinen, and M. Latva-aho, "On the spectral efficiency of full-duplex small cell wireless systems," IEEE Trans. Wireless Commun., vol. 13, no. 9, pp. 4896-4910, Sep. 2014.

[11] H. S. Dhillon, M. Kountouris, and J. G. Andrews, "Downlink MIMO hetnets: Modeling, ordering results and performance analysis," IEEE Trans. Wireless Commun., vol. 12, no. 10, pp. 5208-5222, Oct. 2013.

[12] A. K. Gupta, H. S. Dhillon, S. Vishwanath, and J. G. Andrews, "Downlink multi-antenna heterogeneous cellular network with load balancing," IEEE Trans. Commun., vol. 62, no. 11, pp. 4052-4067, Nov. 2014.

[13] J. Hoydis, K. Hosseini, S. T. Brink, and M. Debbah, "Making smart use of excess antennas: Massive MIMO, small cells, and TDD," Bell Labs Technical Journal, vol. 18, no. 2, pp. 5-21, Sept. 2013.

[14] H. Xie, B. Wang, F. Gao, and S. Jin, "A full-space spectrum-sharing strategy for massive mimo cognitive radio systems," IEEE J. Sel. Areas Commun., vol. 34, no. 10, pp. 2537-2549, Oct. 2016.

[15] H. Xie, F. Gao, S. Zhang, and S. Jin, "A unified transmission strategy for tdd/fdd massive mimo systems with spatial basis expansion model," IEEE Transactions on Vehicular Technology, vol. 66, no. 4, pp. 31703184, Apr. 2017.

[16] C. Li, J. Zhang, J. G. Andrews, and K. B. Letaief, "Success probability and area spectral efficiency in multiuser MIMO hetnets," IEEE Trans. Commun., vol. 64, no. 4, pp. 1544-1556, Apr. 2016.

[17] J. Lee and T. Q. S. Quek, "Hybrid full-/half-duplex system analysis in heterogeneous wireless networks," IEEE Trans. Wireless Commun., vol. 14, no. 5, pp. 2883-2895, May. 2015.

[18] H. Alves, C. H. M. de Lima, P. H. J. Nardelli, R. D. Souza, and M. Latva-aho, "On the average spectral efficiency of interference-limited full-duplex networks," in Proc. CROWNCOM, Jun. 2014, pp. 550-554.

[19] B. Li, D. Zhu, and P. Liang, "Small cell in-band wireless backhaul in massive mimo systems: A cooperation of next-generation techniques," IEEE Trans. Wireless Commun., vol. 14, no. 12, pp. 7057-7069, Dec. 2015.

[20] Y. Li, P. Fan, L. Anatolii, and L. Liu, "On the spectral and energy efficiency of full-duplex small cell wireless systems with massive MIMO," IEEE Trans. Veh. Technol., vol. PP, no. 99, pp. 1-1, 2016

[21] H. Tabassum, A. H. Sakr, and E. Hossain, "Analysis of massive MIMOenabled downlink wireless backhauling for full-duplex small cells," IEEE Trans. Commun., vol. 64, no. 6, pp. 2354-2369, Jun. 2016.

[22] T. K. Vu, M. Bennis, S. Samarakoon, M. Debbah, and M. Latvaaho, "Joint in-band backhauling and interference mitigation in 5G heterogeneous networks," in European Wireless 2016; 22th European Wireless Conference, May 2016, pp. 1-6.

[23] A. AlAmmouri, H. ElSawy, O. Amin, and M. S. Alouini, "In-band $\alpha$ -duplex scheme for cellular networks: A stochastic geometry approach," IEEE Trans. Wireless Commun., vol. 15, no. 10, pp. 6797-6812, Oct. 2016.

[24] A. H. Sakr and E. Hossain, "On cell association in multi-tier fullduplex cellular networks," CoRR, vol. abs/1607.01119, 2016. [Online]. Available: http://arxiv.org/abs/1607.01119

[25] Y. Deng, L. Wang, M. Elkashlan, M. D. Renzo, and J. Yuan, "Modeling and analysis of wireless power transfer in heterogeneous cellular networks," IEEE Transactions on Communications, vol. 64, no. 12, pp. 5290-5303, Dec 2016.

[26] S. Akbar, Y. Deng, A. Nallanathan, M. Elkashlan, and A. H. Aghvami, "Simultaneous wireless information and power transfer in $\mathrm{K}$ tier heterogeneous cellular networks," IEEE Transactions on Wireless Communications, vol. 15, no. 8, pp. 5804-5818, Aug 2016.

[27] A. M. Hunter, J. G. Andrews, and S. Weber, "Transmission capacity of ad hoc networks with spatial diversity," IEEE Trans. Wireless Commun. vol. 7, no. 12, pp. 5058-5071, Dec. 2008.
[28] M. Kountouris and J. G. Andrews, "Downlink SDMA with limited feedback in interference-limited wireless networks," IEEE Trans. Wireless Commun., vol. 11, no. 8, pp. 2730-2741, Aug. 2012.

[29] S. Goyal, C. Galiotto, N. Marchetti, and S. Panwar, "Throughput and coverage for a mixed full and half duplex small cell network," in Proc. IEEE ICC, May 2016, pp. 1-7.

[30] D. Bharadia and S. Katti, "Full duplex MIMO radios," Proc. 11th USENIX Symp. NSDI, pp. 359-372, 2014.

[31] T. D. Novlan, H. S. Dhillon, and J. G. Andrews, "Analytical modeling of uplink cellular networks," IEEE Trans. Wireless Commun., vol. 12, no. 6, pp. 2669-2679, Jun. 2013.

[32] H. ElSawy and E. Hossain, "On stochastic geometry modeling of cellular uplink transmission with truncated channel inversion power control," IEEE Trans. Wireless Commun., vol. abs/1401.6145, 2014.

[33] C. Cox and E. Ackerman, "Demonstration of a single-aperture, fullduplex communication system," in 2013 IEEE Radio and Wireless Symposium, Jan. 2013, pp. 148-150.

[34] L. Wang, K. K. Wong, M. Elkashlan, A. Nallanathan, and S. Lambotharan, "Secrecy and energy efficiency in massive MIMO aided heterogeneous C-RAN: A new look at interference," IEEE J. Sel. Top. Signal Process., vol. 10, no. 8, pp. 1375-1389, Dec. 2016.

[35] A. Ghosh, N. Mangalvedhe, R. Ratasuk, B. Mondal, M. Cudak, E. Visotsky, T. A. Thomas, J. G. Andrews, P. Xia, H. S. Jo, H. S. Dhillon, and T. D. Novlan, "Heterogeneous cellular networks: From theory to practice," IEEE Commun. Mag., vol. 50, no. 6, pp. 54-64, Jun. 2012.

[36] A. He, L. Wang, M. Elkashlan, Y. Chen, and K. K. Wong, "Spectrum and energy efficiency in massive MIMO enabled HetNets: A stochastic geometry approach," IEEE Commun. Lett., vol. 19, no. 12, pp. 22942297, Dec 2015.

[37] A. Shojaeifard, K. K. Wong, M. D. Renzo, G. Zheng, K. A Hamdi, and J. Tang, "Massive MIMO-enabled full-duplex cellular networks," CoRR, vol. abs/1611.03854, 2016. [Online]. Available: http://arxiv.org/abs/1611.03854

[38] S. M. Yu and S. L. Kim, "Downlink capacity and base station density in cellular networks," in 11th International Symposium and Workshops on Modeling and Optimization in Mobile, Ad Hoc and Wireless Networks (WiOpt), May 2013, pp. 119-124.

[39] K. Hosseini, W. Yu, and R. S. Adve, "Large-scale MIMO versus network MIMO for multicell interference mitigation," IEEE J. Sel. Areas Commun., vol. 8, no. 5, pp. 930-941, Oct. 2014.

[40] E. Björnson, J. Hoydis, M. Kountouris, and M. Debbah, "Massive MIMO systems with non-ideal hardware: Energy efficiency, estimation, and capacity limits," IEEE Transactions on Information Theory, vol. 60, no. 11, pp. 7112-7139, Nov 2014.

[41] T. L. Marzetta, "How much training is required for multiuser MIMO?" in Proc. 40th Asilomar Conference on Signals, Systems and Computers, Pacific Grove, CA, Oct. 2006, pp. 359-363.

[42] T. Riihonen, S. Werner, and R. Wichman, "Mitigation of loopback selfinterference in full-duplex MIMO relays," IEEE Trans. Signal Process., vol. 59, no. 12, pp. 5983-5993, Dec. 2011.

[43] A. C. Cirik, Y. Rong, and Y. Hua, "Achievable rates of full-duplex MIMO radios in fast fading channels with imperfect channel estimation," IEEE Trans. Signal Process., vol. 62, no. 15, pp. 3874-3886, Aug. 2014.

[44] H.-S. Jo, Y. J. Sang, P. Xia, and J. G. Andrews, "Heterogeneous cellular networks with flexible cell association: A comprehensive downlink SINR analysis," IEEE Trans. Wireless Commun., vol. 11, no. 10, pp. 34843495, Oct. 2012.

[45] K. Hosseini, W. Yu, and R. S. Adve, "Large-scale MIMO versus network MIMO for multicell interference mitigation," IEEE J. Sel. Areas Commun., vol. 8, no. 5, pp. 930-941, Oct. 2014.

[46] W. C. Cheung, T. Q. S. Quek, and M. Kountouris, "Throughput optimization, spectrum allocation, and access control in two-tier femtocell networks," IEEE J. Sel. Areas Commun., vol. 30, no. 3, pp. 561-574, Apr. 2012.

[47] D. Bethanabhotla, O. Y. Bursalioglu, H. C. Papadopoulos, and G. Caire, "User association and load balancing for cellular massive MIMO," in Information Theory and Applications Workshop (ITA), 2014, Feb. 2014, pp. $1-10$.

[48] M. Haenggi, Stochastic geometry for wireless networks. Cambridge University Press, 2012.

[49] S. Singh, X. Zhang, and J. G. Andrews, "Joint rate and SINR coverage analysis for decoupled uplink-downlink biased cell associations in hetnets," IEEE Trans. Wireless Commun., vol. 14, no. 10, pp. 53605373, Oct 2015

[50] J. G. Wendel, "The non-absolute convergence of Gil-Pelaez' inversion integral," Ann. Math. Statist., vol. 32, no. 1, pp. 338-339, Mar. 1961. 


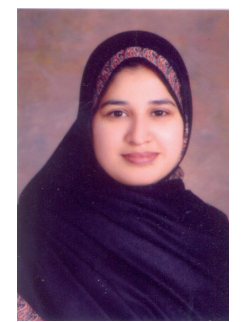

Sunila Akbar (S'15) received the B.E. degree in electrical engineering and the M.Eng. degree in telecommunications engineering from the NED University of Engineering and Technology (NEDUET), Karachi, Pakistan, in 1998 and 2007, respectively. She is currently pursuing the Ph.D. degree with the Department of Informatics, King's College London, U.K. She was a Projects Coordinator with the Electrical Division in a Contracting Company, Dubai, United Arab Emirates, for five years, before joining NEDUET as a Lecturer in 2004. She was appointed as an Assistant Professor with NEDUET in 2008. Her current research interests include statistical modeling of wireless networks, heterogeneous cellular networks, massive MIMO, and energy efficient communications. She has been a Reviewer in several IEEE transactions, letters, and conferences.

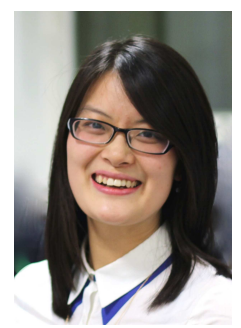

Yansha Deng (S'13-M'17) received her Ph.D. degree in Electrical Engineering from Queen Mary University of London, UK, 2015. From 2015 to 2017, She was the postdoctoral research fellow with the Department of Informatics, at King's College London, UK. She is currently the lecturer in the Department of Informatics, at King's College London, UK. Her research interests include massive MIMO, HetNets, molecular communication, cognitive radio, cooperative networks, and physical layer security. She has received the Best Paper Award in ICC 2016. She is currently an Editor of IEEE Communications Letters. She has also served as TPC member for many IEEE conferences, such as IEEE GLOBECOM and ICC.

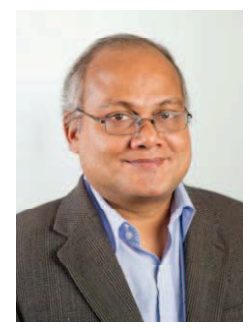

Arumugam Nallanathan (S'97-M'00-SM'05-F'17) is Professor of Wireless Communications in the School of Electronic Engineering and Computer Science at Queen Mary University of London since September 2017. He was with the Department of Informatics at King's College London from December 2007 to August 2017, where he was Professor of Wireless Communications from April 2013 to August 2017. He was an Assistant Professor in the Department of Electrical and Computer Engineering, National University of Singapore from August 2000 to December 2007. His research interests include 5G Wireless Networks, Internet of Things (IoT) and Molecular Communications. He published more than 350 technical papers in scientific journals and international conferences. $\mathrm{He}$ is a co-recipient of the Best Paper Award presented at the IEEE International Conference on Communications 2016 (ICC2016) and IEEE International Conference on Ultra-Wideband 2007 (ICUWB 2007). He is an IEEE Distinguished Lecturer. He has been selected as a Web of Science (ISI) Highly Cited Researcher in 2016. He is an Editor for IEEE Transactions on Communications and IEEE Transactions on Vehicular Technology. $\mathrm{He}$ was an Editor for IEEE Transactions on Wireless Communications (20062011), IEEE Wireless Communications Letters and IEEE Signal Processing Letters. He served as the Chair for the Signal Processing and Communication Electronics Technical Committee of IEEE Communications Society and Technical Program Chair and member of Technical Program Committees in numerous IEEE conferences. He received the IEEE Communications Society SPCE outstanding service award 2012 and IEEE Communications Society RCC outstanding service award 2014.

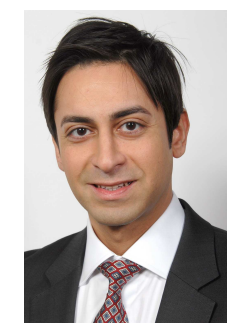

Maged Elkashlan (M'06) received the Ph.D. degree in Electrical Engineering from the University of British Columbia, Canada, 2006. From 2007 to 2011, he was with the Commonwealth Scientific and Industrial Research Organization (CSIRO), Australia. During this time, he held visiting appointments at University of New South Wales and University of Technology Sydney. In 2011, he joined the School of Electronic Engineering and Computer Science at Queen Mary University of London, UK. His research interests fall into the broad areas of communication theory and statistical signal processing.

Dr. Elkashlan currently serves as Editor of IEEE TRANSACTIONS ON WIRELESS COMMUNICATIONS and IEEE TRANSACTIONS ON VEHICULAR TeChnology. He received the Best Paper Award at the IEEE International Conference on Communications (ICC) in 2016 and 2014, the International Conference on Communications and Networking in China (CHINACOM) in 2014, and the IEEE Vehicular Technology Conference (VTC-Spring) in 2013.

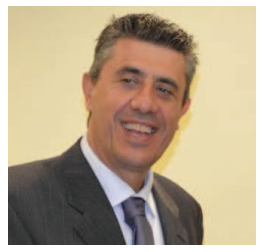

George K. Karagiannidis (M'96-SM'03-F'14) was born in Pithagorion, Samos Island, Greece. He received the University Diploma (5 years) and $\mathrm{PhD}$ degree, both in electrical and computer engineering from the University of Patras, in 1987 and 1999, respectively. From 2000 to 2004, he was a Senior Researcher at the Institute for Space Applications and Remote Sensing, National Observatory of Athens, Greece. In June 2004, he joined the faculty of Aristotle University of Thessaloniki, Greece where he is currently Professor in the Electrical \& Computer Engineering Dept. and Director of Digital Telecommunications Systems and Networks Laboratory. He is also Honorary Professor at South West Jiaotong University, Chengdu, China. His research interests are in the broad area of Digital Communications Systems and Signal processing, with emphasis on Wireless Communications, Optical Wireless Communications, Wireless Power Transfer and Applications, Molecular and Nanoscale Communications, Stochastic Processes in Biology and Wireless Security. He is the author or co-author of more than 400 technical papers published in scientific journals and presented at international conferences. He is also author of the Greek edition of a book on "Telecommunications Systems" and co-author of the book "Advanced Optical Wireless Communications Systems", Cambridge Publications, 2012. Dr. Karagiannidis has been involved as General Chair, Technical Program Chair and member of Technical Program Committees in several IEEE and non-IEEE conferences. In the past, he was Editor in IEEE Transactions on Communications, Senior Editor of IEEE Communications Letters, Editor of the EURASIP Journal of Wireless Communications \& Networks and several times Guest Editor in IEEE Selected Areas in Communications. From 2012 to 2015 he was the Editor-in Chief of IEEE Communications Letters. Dr. Karagiannidis is IEEE Fellow and one of the highly-cited authors across all areas of Electrical Engineering, recognized as 2015 and 2016 Thomson Reuters highly-cited researcher. 